\title{
Dust environment of an airless object: A phase space study with kinetic models
}

\author{
Kallio, E1)., S. Dyadechkin, S. Fatemi, M. Holmström, Y. Futaana, \\ P. Wurz, V. A. Fernandes, F. Álvarez, J. Heilimo. R. Jarvinen, W. Schmidt, A.-M. Harri, \\ S. Barabash, J. Mäkelä, N. Porjo and M. Albo \\ 1) Aalto University, School of Electrical Engineering, email: esa.kallio@aalto.fi; phone: +358 504205857
}

\begin{abstract}
The study of dust above the lunar surface is important for both science and technology. Dust particles are electrically charged due to impact of the solar radiation and the solar wind plasma and, therefore, they affect the plasma above the lunar surface. Dust is also a health hazard for crewed missions because micron and sub-micron sized dust particles can be toxic and harmful to the human body. Dust also causes malfunctions in mechanical devices and is therefore a risk for spacecraft and instruments on the lunar surface.
\end{abstract}

Properties of dust particles above the lunar surface are not fully known. However, it can be stated that their large surface area to volume ratio due to their irregular shape, broken chemical bonds on the surface of each dust particle, together with the reduced lunar environment cause the dust particles to be chemically very reactive. One critical unknown factor is the electric field and the electric potential near the lunar surface. We have developed a modelling suite, Dusty Plasma Environments: near-surface characterization and Modelling (DPEM), to study globally and locally dust environments of the Moon and other airless bodies. The DPEM model combines three independent kinetic models: (1) a 3D hybrid model, where ions are modelled as particles and electrons are modelled as a charged neutralizing fluid, (2) a 2D electrostatic Particle-in-Cell (PIC) model where both ions and electrons are treated as particles, and (3) a 3D Monte Carlo (MC) model where dust particles are modelled as test particles. The three models are linked to each other unidirectionally; the hybrid model provides upstream plasma parameters to be used as boundary conditions for the PIC model which generates the surface potential for the MC model.

We have used the DPEM model to study properties of dust particles injected from the surface of airless objects such as the Moon, the Martian moon Phobos and the asteroid RQ36. We have performed a $\left(v_{0}\right.$, $m / q)$-phase space study where the property of dust particles at different initial velocity $\left(v_{o}\right)$ and initial mass per charge $(\mathrm{m} / \mathrm{q})$ ratio were analysed. The study especially identifies regions in the phase space where the electric field within a non-quasineutral plasma region above the surface of the object, the Debye layer, becomes important compared with the gravitational force. Properties of the dust particles in the phase space region where the electric field plays an important role are studied by a 3D Monte Carlo model.

The current DPEM modelling suite does not include models of how dust particles are initially injected from the surface. Therefore, the presented phase space study cannot give absolute 3D dust density distributions around the analysed airless objects. For that, an additional emission model is necessary, which determines how many dust particles are emitted at various places on the analysed ( $v_{0}, \mathrm{~m} / \mathrm{q}$ )phase space. However, this study identifies phase space regions where the electric field within the Debye layer plays an important role for dust particles. Overall, the initial results indicate that when a realistic dust emission model is available, the unified lunar based DPEM modelling suite is a powerful tool to study globally and locally the dust environments of airless bodies such as planetary moons, Mercury, asteroids and non-active comets far from the Sun. 


\section{Introduction}

The Moon is the best known example of the so-called direct plasma-surface interaction where plasma interacts directly with the surface of the object. This direct interaction takes place at the lunar surface because the Moon has neither an atmosphere nor a global intrinsic magnetic field, which could change the motion of charged particles near the surface. Thus, the Moon is an ideal object to study various physical processes near the surface, which are anticipated to occur on the so-called airless bodies, like asteroids, other planetary moons in the Solar System and non-active dusty comets.

One can anticipate that many physical parameters affect surface processes near the lunar surface (see e.g. Kallio et al., 2012, and discussion therein): (1) the density, bulk velocity and temperature of the solar wind protons and electrons; (2) secondary particles (electrons, positively and negatively charged ions) resulting from the impact of the solar wind onto the surface; (3) photoelectrons from the surface in places exposed to the sunlight; (4) charged dust particles above the surface, which are also sinks and sources of charged particles like the surface itself; (5) the interplanetary magnetic field (IMF), the magnetic field associated with the Earth's magnetosheath or with the magnetosphere. Furthermore, possible local magnetic anomalies affect the properties of plasma, for example, reflection of the solar wind electrons and protons from and above the surface requiring e.g. hybrid modelling beyond the Debye scale; (6) the convective electric field associated with the flow of the solar wind, with the electric field in the Earth's magnetosheath or in the magnetosphere (e.g. Kallio and Facskó, 2015). Moreover, there is an electric field within the Debye sheath (or the Debye layer) where the plasma is not quasineutral. Furthermore, the solar radiation varies due to temporal variations of the Sun. The intensity of the solar radiation at a given point on the lunar surface varies also with the orbit of the Moon around the Earth. Moreover, physical and chemical properties of the locations on the lunar surface from where the charged particles originate vary (e.g., mafic basalt flows or crustal anorthositic material) vary. Finally, surface processes are affected by topographical variations due to lunar landscapes (e.g. Dyadechkin et al., 2015).

In addition to the aforementioned physical parameters and processes the lunar near-surface is highly important for basic space plasma physics research. Such research is also needed to improve the understanding of the interaction between the dust particles on the lunar surface and the Debye layer that directly affect the technical and scientific instrumentation deployed on the lunar surface during different missions. Ultimately, these effects can pose potential hazard to humans (e.g. Linnarsson et al., 2012). In addition, laboratory experiments have shown that due to the electric field near the surface the dust particles can easily be displaced [Wang et al., 2010] potentially causing malfunctions of moving spacecraft parts and space instruments existing on the lunar surface. Moreover, dust can contaminate astronomical observations in the infra-red, visible and UV wavelength ranges [Murphy and Vondrak, 1993; Stubbs et al., 2006]. Lunar dust has also been observed far above the surface (see e.g. references in Stubbs et al., 2006) suggesting that dust may have global effects.

Space weathering caused by micrometeorites, galactic cosmic rays and solar energetic particles erode, and vaporize dust grains and regolith on the lunar surface (see e.g. Jordan et al., 2015). However, the difference between dust on the Earth and on the Moon is that on the Moon there is no Earth-like wind or water erosion of dust particles. Therefore, small lunar dust particles can have very sharp edges and reactive broken chemical bonds (e.g. Liu and Taylor, 2011; Liu et al., 2008; Linnarsson et al., 2012). When entered into the lungs of an astronaut, the small sharp dust particles are therefore a potential health hazard. Generally speaking, lunar dust is more chemically reactive, has large surface areas, and is 
composed of sharper jagged edges than Earth's dust [Cain, 2010]. Properties of dust are therefore a critical issue that has to be taken into account when a crewed lunar mission is planned.

A comprehensive lunar dust model should consider three different space regimes. It should include (1) a global model that gives properties of the solar wind plasma impacting on the surface, (2) a local model of the electric field above the lunar surface which accelerates dust particles, and (3) a global model which gives the density of dust around the Moon. Towards this goal, the present study focuses on three different but connected models. A global hybrid model gives the properties of protons above the surface. A local 2D full kinetic model is then used to derive the surface potential at different Solar Zenith Angles (SZA) near the surface. Finally, a global Monte Carlo model is used to derive three dimensional densities of the dust particles escaping from the surface. The comprehensive lunar dust model is then applied to study the 3D dust density profiles for the Moon, the Martian moon Phobos and the asteroid RQ36 to cover a large size range of planetary objects.

The paper is organized as follows. First the three developed models are described. The capability of the models is demonstrated with a phase space study where the models are used to derive 3D dust densities for the Moon, Phobos and the asteroid RQ36 for parameters entered manually and for two different dust emission models: a homogeneous dust emission for the surface, and a point source at the subsolar point. For parameter evaluation, initial dust particles were chosen from a range that makes it possible to analyse effects of the surface electric potential and, consequently, the capacity of the developed modelling suite to study various airless bodies. Finally, lessons of the analysed cases and a roadmap for future more sophisticated dust models are discussed.

\section{Description of the Dusty Plasma Environments: near-surface characterization and Modelling (DPEM) suite}

The DPEM model contains three kinetic models: (1) a 3D Hybrid model where ions are particles and electrons form a fluid, (2) a 2D electrostatic full kinetic Particle-in-Cell (PIC) model where both ions and electrons are particles, and (3) a 3D Monte Carlo (MC) model.

Figure 1 shows an illustration of the models and depicts how they are connected to each other by unidirectional connections where the hybrid model provides the plasma parameter boundary conditions for the PIC model, which in turn gives inputs to the MC model. More precisely, the hybrid model provided the positions and velocities of the solar wind protons which hit the surface of the planetary object. The PIC model uses these particles and derives the macroscopic plasma parameters associated with the precipitating protons: the density of protons, $\left(n\left(\mathrm{H}^{+}\right)\right)$, three bulk velocity components for protons $\left(U_{x}\left(\mathrm{H}^{+}\right), U_{y}\left(\mathrm{H}^{+}\right), U_{z}\left(\mathrm{H}^{+}\right)\right)$and the temperatures of protons in three directions $\left(T_{x}\left(\mathrm{H}^{+}\right), T_{y}\left(\mathrm{H}^{+}\right)\right.$, $\left.T_{2}\left(\mathrm{H}^{+}\right)\right)$. Here the indices refer to the Object-centred Solar wind Orbital (OSO) coordinates where the $\mathrm{x}$-axis points from the centre of the (spherical) object against the flow of the solar wind, the $y$-axis shows the direction of the velocity vector of the object perpendicular the $x$-axis, and the $z$-axis completes the right handed coordinate system. All three models include their own 3D Maxwellian Particle Generator, MPG, which are used to inject particles into models. If the macroscopic parameters associated with the precipitating protons are close to the values of the undisturbed solar wind parameters, as in the cases analysed in this study as discussed later in Section 2.1, the PIC model generates particles by using its own MPG which uses undisturbed plasma parameters. Finally, the PIC model gives the surface potential values to the MC model which uses the potential values in its MPG as will be discussed later in detail in Section 2.3.2. 
All three models in the DPEM suite are kinetic simulations where some, or all, of the particle species are modelled as particles. The particles are propagated by using Newton's second law:

$$
\begin{aligned}
& m \frac{d \boldsymbol{v}(\boldsymbol{r}, t)}{d t}=m \boldsymbol{g}(\boldsymbol{r})+q \boldsymbol{E}(\boldsymbol{r}, t)+q \boldsymbol{v} \times \boldsymbol{B}(\boldsymbol{r}, t)+\boldsymbol{F} \\
& \frac{d \boldsymbol{r}(\boldsymbol{r}, t)}{d x}=\boldsymbol{v}(\boldsymbol{r}, t)
\end{aligned}
$$

Here, $q, m$ and $\boldsymbol{v}$ are the charge, mass and velocity of a particle, $\boldsymbol{g}$ is the gravitation acceleration at the point where the particle is, $\boldsymbol{E}$ is the electric field, $\boldsymbol{B}$ the magnetic field and $\boldsymbol{F}$ is the contribution of all other forces, such as caused by the radiation pressure or collisions. The three models differ from each other in the way particles are modelled, how the electric field is derived and how the magnetic field is treated.

The objects were assumed to be spherical balls with a homogeneous mass density end electrical conductivity. However, the models do not include information about the detailed chemical composition of the surface material. The DPEM model was used to study three different objects of different sizes, masses and escape velocities:

\section{Object No1: Near Earth asteroid RQ36}

The RQ36, or 101955 Bennu, is an Apollo near Earth asteroid discovered in 1999. In the simulations RQ36 in the solar wind was assumed to have a radius of $252 \mathrm{~m}$ and a mass of $1.4 \times 10^{11} \mathrm{~kg}$. The escape velocity from RQ36 is about $0.2 \mathrm{~m} / \mathrm{s}$.

\section{Object No2: Martian moon Phobos}

Phobos was assumed to have radius of $12 \mathrm{~km}$ and the mass of $1.066 \times 10^{16} \mathrm{~kg}$. The escape velocity from Phobos is about $11 \mathrm{~m} / \mathrm{s}$ and the surface gravity is $0.0057 \mathrm{~m} / \mathrm{s}^{2}$. In the simulations Phobos was assumed to be in the solar wind.

\section{Object No3: The Moon.}

In the global simulations the Moon was assumed to be in the solar wind, and to have a radius of $1730 \mathrm{~km}$ and a mass of $7.35 \times 10^{22} \mathrm{~kg}$. The escape velocity from the Moon is about $2.5 \mathrm{~km} / \mathrm{s}$ and the surface gravity is $1.622 \mathrm{~m} / \mathrm{s}^{2}$.

Moreover, in the PIC model the EUV light is directed along the $x$-axis, which results in photo-electron emission from the surface of the planetary body. Photo-electrons were modelled by a 3D Maxwellian velocity distribution function with the thermal velocity of $621 \mathrm{~km} / \mathrm{s}$, which corresponds to a temperature of about $2.2 \mathrm{eV}$. The photo-electron emission current density was assumed to be $4.5 \mu \mathrm{Am}^{-}$ ${ }^{2}$ based on measurements [Willis et al., 1973] and which has been used in previous 1D PIC simulations (see, e.g., Poppe and Horànyi, 2010). Moreover, the high energy tail of the Maxwellian velocity distribution function was cut away by removing electrons above $6 \mathrm{eV}$.

\subsection{Hybrid model}

In the hybrid model, ions are modelled as particles according to Eqs. 1-2. Electrons are modelled as a massless fluid. The charge density of electrons, $n_{e}$, is assumed to be equal to the total charge density of positively charged ions, that is, the plasma is assumed to be quasi-neutral. In the DPEM hybrid model the only positively charged ions were the solar wind protons. The electric field is derived from the electron momentum equation and the definition of the electric current: 


$$
\begin{aligned}
& \boldsymbol{E}(\boldsymbol{r}, t)=-\boldsymbol{U}_{e}(\boldsymbol{r}, t) \times \boldsymbol{B}(\boldsymbol{r}, t) \\
& \boldsymbol{U}_{e}(\boldsymbol{r}, t)=\boldsymbol{U}_{H^{+}}(\boldsymbol{r}, t)-\boldsymbol{j}(\boldsymbol{r}, t) / e n_{e}(\boldsymbol{r}, t)
\end{aligned}
$$

Here $\boldsymbol{U}_{e}, \boldsymbol{U}_{H^{+}}, \boldsymbol{j}$ and $e$ are the electron bulk velocity, bulk velocity of protons, the electric current, and the positive unit charge, respectively (see e.g. Kallio, 2005, for the details of a 3D hybrid model simulations for a Moon-type of airless objects without an intrinsic magnetic field). The object is assumed to be an insulator (resistivity $1 \times 10^{7} \mathrm{ohm} / \mathrm{m}$ ) into which the IMF can diffuse (see Holmström et al., 2012, for the details of the model). The upstream parameters were $n\left(\mathrm{H}^{+}\right)=7.1 \mathrm{~cm}^{-3}, \boldsymbol{U}\left(\mathrm{H}^{+}\right)=$ $[-450,0,0] \mathrm{km} / \mathrm{s}, T\left(\mathrm{H}^{+}\right)=1.2 \times 10^{5} \mathrm{~K}$ and the IMF was $\boldsymbol{B}=[7 / \sqrt{ } 2,7 / \sqrt{ } 2,0] \mathrm{nT}$.

Figure 2 shows a snapshot of the magnetic field magnitude obtained from the hybrid model run for the Moon. In the run the grid cell size was $200 \times 200 \times 200 \mathrm{~km}^{3}$. The simulation domain size is $12000 \mathrm{~km}$ along the $\mathrm{x}$-axis, and $8000 \mathrm{~km}$ along the $y$-and z-axis. An important feature in the Moon case is that the IMF diffuses into the body and no bow shock is formed upstream of the object. Therefore, the solar wind is practically undisturbed on the dayside $\left(\mathrm{SZA}<90^{\circ}\right)$. On the other hand, on the nightside of the Moon a long magnetic wake is formed, as seen in Fig. 2, and this region $\left(\mathrm{SZA}>90^{\circ}\right.$ ) is highly distorted.

\subsection{Electrostatic PIC model}

In the electrostatic PIC simulation both ions and electrons are modelled as particles. In contrast to the hybrid model the plasma is not assumed to be quasi-neutral and, consequently, charge separation can occur. In the PIC model the external force $\boldsymbol{F}$ in Eq. 1a includes only the gravitational force. The electric field is derived from the electric potential (Eq. 3), $\varphi$, which in turn is obtained from the total electric charge density, $\rho_{q}$, from the Poisson's equation (Eq. 4):

$$
\begin{aligned}
& \boldsymbol{E}(\boldsymbol{r}, t)=-\nabla \varphi(\boldsymbol{r}, t) \\
& \nabla^{2} \varphi(\boldsymbol{r}, t)=-\rho_{q}(\boldsymbol{r}, t) / \epsilon_{o}
\end{aligned}
$$

Here $\epsilon_{o}$ is the electric permittivity of free space. As already mentioned, in the runs presented in this paper the PIC model was electrostatic, that is, the magnetic field was not assumed to have a time dependency. Moreover, in the analysed PIC simulations the magnetic field was assumed to be zero. The boundary condition on the solar wind wall at $x=100 \mathrm{~m}$ is that the electric field is zero, therefore, the electric potential is a constant. The length of the cell was $0.25 \mathrm{~m}$.

The architecture of the DPEM model enables injection of the protons that have impacted the surface in the hybrid model into the PIC model. The properties of the precipitating protons were tested by examining with the hybrid model when density, bulk velocity and thermal velocity of the solar wind were $7.1 \mathrm{~cm}^{-3},[-450,0,0] \mathrm{km} / \mathrm{s}$ and $1.2 \times 10^{5} \mathrm{~K}$, respectively. The IMF was assumed to be [-4.95, $+4.95,0] \mathrm{nT}$. The plasma density, $n$, three bulk velocity components, $\left(U_{x}, U_{y}, U_{*}\right)$, and three temperature components, $\left(T_{x}, T_{y}, T_{\partial}\right)$, were derived for the three planetary objects by using the algorithms (see. e.g. analysis in Kallio et al., 1997)

$$
n=\frac{R}{d T d A} \sum_{i} \frac{w_{i}}{\left|\boldsymbol{v}_{i} \cdot \boldsymbol{r}_{i}\right|}
$$




$$
\begin{gathered}
\boldsymbol{U}=\sum_{i} \boldsymbol{v}_{i} \frac{w_{i}}{\left|\boldsymbol{v}_{i} \cdot \boldsymbol{r}_{i}\right|} / \sum_{i} \frac{w_{i}}{\left|\boldsymbol{v}_{i} \cdot \boldsymbol{r}_{i}\right|} \\
T_{k}=\frac{m_{p}}{k_{B}} \sum_{i}\left(\boldsymbol{v}_{k i}-\boldsymbol{U}_{k}\right)^{2} \frac{w_{i}}{\left|\boldsymbol{v}_{i} \cdot \boldsymbol{r}_{i}\right|} / \sum_{i} \frac{w_{i}}{\left|\boldsymbol{v}_{i} \cdot \boldsymbol{r}_{i}\right|}
\end{gathered}
$$

Here $R$ is the radius of the planetary object, $\boldsymbol{r}_{i}=\left(x_{i}, y_{i}, z_{i}\right)$ is the position vector where the ion $i$ hits the surface, $\boldsymbol{v}_{i}=\left(v_{x i}, v_{j i}, v_{i i}\right)$ : the velocity vector of an ion $i$ which hits the sphere, $d A_{\text {collected }}$ is the area on the planetary sphere from where the particles were collected on the surface, $d T_{\text {collected }}$ is the particle accumulation time, $m_{p}$ is the mass of a proton and $k_{B}$ is the Boltzmann's constant. The sum is over all particles that hit the surface $d A_{\text {collected }}$ during a time interval $d T_{\text {collected }}$. The subscript $k$ in Eq. $5 \mathrm{c}$ shows the component of the velocity, i.e. $x, y$ or $z$. The particles were collected in $10^{\circ} \mathrm{SZA}$ angle bins from SZA $=0^{\circ}$ up to the SZA where the last hit on the surface was recorded. For the Moon, Phobos and asteroid RQ36 $d T_{\text {collected }}$ was $50 \mathrm{~s}, 10 \mathrm{~s}$ and $0.3226 \mathrm{~s}$, respectively.

An important feature of the derived parameters was that the plasma parameters on the dayside were much like in the undisturbed solar wind. Another important feature is that there were no hits of $\mathrm{H}^{+}$ ions on the surface of the Moon, Phobos and RQ36 at SZA values larger than $\sim 107^{\circ}, \sim 105^{\circ}$ and $\sim 105^{\circ}$, respectively (c.f. Fig. 3d). This limitation is related to the fact that the hybrid model assumes quasi-neutrality while in reality a charge separation takes place on the nightside which results in an ambipolar electric field accelerating protons deep into the tail (see e.g. Kallio et al., 2005, and references therein). Moreover, the number of precipitating particles decrease rapidly at SZA $>90^{\circ}$ which causes statistical fluctuations to the estimation of the particle density (Fig. 3a) and particle temperature (Fig. 3c). This result indicates that the hybrid model cannot give accurate plasma parameters on the nightside near the surface for the PIC simulation. Therefore, only the dayside region is analysed in this study with the PIC model and the plasma is assumed to be undisturbed. Note that non-disturbed plasma parameters on the surface are, indeed, expected to exist because objects in the hybrid model absorb particles and let the IMF diffuse inside them without the formation of an obstacle or a bow shock above the surface (c.f. Fig. 2).

Localised 2D PIC simulations were made for six different SZA values $\left(0^{\circ}, 15^{\circ}, 30^{\circ}, 45^{\circ}, 60^{\circ}\right.$ and $\left.75^{\circ}\right)$ assuming that the surface is a plane. The Moon is assumed to be in the nominal solar wind where density, bulk velocity, and kinetic temperature of the undisturbed solar wind protons were $10 \mathrm{~cm}^{-3}, 400$ $\mathrm{km} / \mathrm{s}$ and $10 \mathrm{eV}$, respectively. The bulk velocity of electrons was also $400 \mathrm{~km} / \mathrm{s}$. The thermal velocity of electrons was $1.36 \times 10^{3} \mathrm{~km} / \mathrm{s}$, which corresponds to temperatures of about $10.5 \mathrm{eV}$. The density of the solar wind electrons was adjusted to have quasi-neutrality on the solar wind wall. The SZA effect was taken into account by multiplying the solar wind velocity and the flux of the photoelectrons by $\cos (S Z A)$, i.e. taking into account the velocity component normal to the surface.

Fig. 4 shows the electric potential in the analysed cases. All potentials are non-monotonic and have local minimum values when the electric field changes its direction. The electric field points upward/downward below/above the local minimum, respectively. This means that positively charged particles are accelerated upward just above the surface and downward near the solar wind wall in the simulation. The most important parameter from the $\mathrm{MC}$ modelling point of view is the surface potential. As seen in Fig. 4, the surface potential decreases with increasing SZA. Moreover, in the analysed cases the surface potential is positive at least up to SZA $=60^{\circ}$ and becomes negative between $\mathrm{SZA}=60^{\circ}-75^{\circ}$. The density of photoelectrons, solar wind electrons, and protons as well as technical details about the developed PIC code can be found in the companion paper [Dyadechkin et al., 2015]. 
It is important also to note that in this study the dust particles were assumed to be positively charged. As can be seen in the PIC model (Fig. 4), the electric field points upward at the planetary surface in all analysed SZA values. Moreover, taking into account (1) the boundary condition $\boldsymbol{E}=0$ at the solar wind wall $(x=100 \mathrm{~m})$, and (2) the assumption that there is no electric charge outside of the simulation box, Gauss' law states, that the total electric charge within the PIC simulation box has to be zero. In this situation an upward pointing electric field on the surface indicates that the surface is positively charged. The dust particles in the MC simulation are originating from the surface. Due to EUV light the dust particle surfaces emit photoelectrons similarly as other surface regions of the object and in this study the dust particles were assumed to be positively charged in the MC model.

\subsection{Monte Carlo model}

In the Monte Carlo model the motion of a dust particle of a mass $m_{d u s t}$, an electric charge of $q_{d u s t}$, and an initial velocity is influenced by a prescribed force field. In the cases analysed in this paper the forces were the Lorentz force $q \boldsymbol{E}+q \boldsymbol{v} \times \boldsymbol{B}$ (c.f. Eq. 1a) and the gravitational force. In the Moon and asteroid RQ36 simulations the IMF was assumed to be $[-4.95,+4.95,0.0] \mathrm{nT}$ and in the Phobos simulations $[-1.63,+2.52,0.0] \mathrm{nT}$. The convection electric field was set to zero to investigate change of the energy of the dust particles caused by the surface charging and gravitation.

The density of the dust was derived from two Monte Carlo models: (1) Dust within and near the Debye layer is studied by a local 2D MC model and the (2) global dust distribution around an object by a 3D MC model. Two different models were used to obtain a good spatial resolution near the surface, where the length scale of the electric potential is the Debye length, and around the object, where the length scale is large, on the order of the radius of an object.

As will be discussed in detail later in Section 2.3.5., it is not known how large the amount of detached dust particles from the surface can become and what might be their masses, electric charges and the velocity distribution functions. Dust particles can be envisioned to be lifted from the surface by micrometeoroid impacts or by a human activity such as walking, driving a lunar rover or landing of a spacecraft. Dust lifting by electrostatic forces has also been a topic of numerous studies (see e.g. Farrell et al., 2007). The particle flux of dust from the surface is also an unknown parameter.

Because of the aforementioned uncertainties several choices have been made in the MC simulations to study the sensitivity of the results to the input parameters. First, simulations were made for different masses, charges and velocities of dust particles. Second, the role of the planetary surface charge, which affects the acceleration of charged dust particles, was studied by making runs with and without surface charging. Third, two different dust source models were analysed: A full surface dust emission case and a point source dust emission case. Fourth, the density of the dust is given in normalized values because the particle flux of dust is not known. Fifth, the initial velocity distribution function of dust particles emitted from the surface was assumed to be Maxwellian.

\subsubsection{Debye layer region}

Fig. 5 shows 1D density profiles of dust particles within the Debye layer in the analysed "cold" and "hot" cases. In the 2D MC runs the dust particles had the mass of $6.2832 \times 10^{-18} \mathrm{~kg}\left(\sim 3.76 \times 10^{9} \mathrm{amu}\right.$, where amu is the atomic mass unit, about the same as the mass of a proton of $1.67 \times 10^{-27} \mathrm{~kg}$ ), and the electric charge of $+3.5322 \times 10^{-17} \mathrm{C}\left(\sim 221 \mathrm{e}\right.$, where e is the positive unit charge of $\left.1.602 \times 10^{-19} \mathrm{C}\right)$. The adopted dust mass and electric charge has the same order of magnitude as the dust $\left(\sim 5.3 \times 10^{8}\right.$ amu, 
$\sim 105$ e) which has recently been used to study the dust density above $2 \mathrm{D}$ lunar surfaces. The adopted mass and charge values correspond to a dust particle with a radius of $\sim 0.05 \mu \mathrm{m}$ and a potential of $\sim 3 \mathrm{~V}$ [Dyadechkin et al., 2015].

Densities were derived for the six electric potential cases shown earlier in Fig. 4. The densities from the 2D MC model given in Fig. 5 are derived along the $x$-axis at $y=0 \mathrm{~m}$. In the 2D PIC model, the $x$-axis is the vertical axis pointing away from the surface, and the $y$-axis is the horizontal axis. Initial mass for the dust were chosen to give an example of the situation where the role of the electric potential can be identified in dust densities. The simulated region was $x=[0,100] \mathrm{m}$, the grid size was $0.25 \mathrm{~m}$, the time step was $0.01 \mathrm{~s}$ and the duration of the simulation was $50 \mathrm{~s}$.

The "cold" dust run is shown in Fig. 5a (the Run PIC-1). In this run dust was launched from the surface with a 3D Maxwellian velocity distribution function with a bulk velocity of $10 \mathrm{~m} / \mathrm{s}$ and thermal velocity of $1 \mathrm{~mm} / \mathrm{s}$. In this "cold" dust case, or a "beam" dust case, the adopted thermal velocity was therefore much smaller than the bulk dust velocity and the maximum dust density value can be found at the altitude where the dust upward moving velocity changes its direction to downward velocity.

Fig. 5b represents the "hot" dust case (the Run PIC-2) where the thermal velocity of $2 \mathrm{~m} / \mathrm{s}$ is comparable with the bulk velocity of $10 \mathrm{~m} / \mathrm{s}$. Thermal spread results in the situation where dust particles turn back to surface at different altitudes resulting in a spreading of the dust density around the maximum dust density. In the "hot" dust case, as in the "cold" dust case, the altitude of the maximum dust density decreases with increasing SZA because the surface charging decreases with the increasing SZA.

Note that the density differences in Fig. $5 \mathrm{a}$ and $5 \mathrm{~b}$ are caused purely by the Debye layer. In the "cold" dust case the maximum density forms a sharp peak because all emitted dust particles had quite similar initial velocities. The maximum altitude is highest at small SZA values because the positive surface potential is largest there and, consequently, it gives the maximum upward pointing acceleration for a positively charged dust particle. The SZA $=60^{\circ}$ case resembles most of the cases without the Debye layer because in this SZA situation the surface potential in near zero (c.f. Fig. 4) and, therefore, it has only slightly increased the maximum altitude from about $31 \mathrm{~m}\left(=0.5 \times(10 \mathrm{~m} / \mathrm{s})^{2} / 1.622 \mathrm{~m} / \mathrm{s}^{2}\right)$, which the dust particles would have had due to the lunar gravitational field alone. The $60^{\circ}$ case also divides the dust density curves into two regions with respect to the SZAs: The SZA case where the (positive) Debye layer increases the maximum altitude and the high SZA case where the maximum altitude is decreased by the Debye layer.

\subsubsection{D MC model}

The 3D dust density distribution function cannot be derived with the similar small grid size as shown in Fig. 5 because of the high computational cost. Therefore, the role of the surface charge was taken into account in the following way. First, the Debye layer region was removed from the 3D MC simulation region. Second, the effect of the removed Debye layer was taken into account by modifying the radial (upward) velocity of a dust particle which is launched in the MC model at SZA, $v_{r}^{M C}(S Z A)$, by using the surface potential at the launch point, $\varphi_{\text {surf }}^{P I C}(S Z A)$, which is derived from the PIC model:

$$
\begin{gathered}
\frac{1}{2} m_{\text {dust }} v_{r}^{M C}(S Z A)^{2}+m_{\text {dust }} U(h)_{g}=\frac{1}{2} m_{\text {dust }} v_{r}^{\text {Maxwell }^{2}}+m_{\text {dust }} U(0)_{g}+q_{\text {dust }} \varphi_{\text {surf }}^{\text {PIC }}(S Z A)[6] \\
=>v_{r}^{M C}(S Z A)=v_{r}^{\text {Maxwell }} \sqrt{1+\frac{2\left(\frac{q_{\text {dust }}}{m_{\text {dust }}}\right) \varphi_{\text {surf }}^{\text {PIC }}(S Z A)-2\left[U(h)_{g}-U(0)_{g}\right]}{v_{r}^{\text {Maxwell }^{2}}}}
\end{gathered}
$$


Here $v_{r}^{\text {Maxwell }}, U(h)_{g}, U(0)_{g}$ are respectively the radial velocity component derived from the $3 \mathrm{D}$ Maxwellian velocity distribution generator of the MC model, the gravitation potential at the height $h$ from the surface, and the gravitational potential on the surface. The two velocity components along the surface were unchanged by the electric potential. As pointed out before, the properties of precipitating protons at the dayside in all three analysed objects were much like the undisturbed solar wind protons on the dayside. Therefore, similar SZA dependent surface potentials were used for all objects at SZA < $90^{\circ}$ as shown in Fig. 4. At higher SZA values the surface potential was assumed to be zero because the hybrid model does not give accurate plasma parameters near the surface, as also noted before.

It is worth recalling how the surface potential affects the radial velocity in Eq. (7). As discussed in Section 2.2, the surface as well as the dust particles can be regarded as positively charged at SZA $<60^{\circ}$. However, a dust particle can also be non-charged because of the low surface charging, as will be discussed in detail later in Section 2.3.5. A situation where a dust particle is positively charged means that the term $q_{\text {dust }} \varphi_{\text {surf }}^{P I C}(S Z A)$ in Eq. (7) is positive at SZA $<60^{\circ}$ and, consequently, that a dust particle which has passed through the Debye layer has increased its radial velocity. This also means that the surface potential increases the number of dust particles, which can escape from the Debye layer because low velocity dust particles can get enough energy to move to high altitudes in the gravitational field. At SZA $=75^{\circ}$, instead, the surface potential is negative and a positively charged dust particle which has passed through the Debye layer has decreased its radial velocity. Therefore the surface potential decreases the number of small velocity dust particles which can escape from the Debye layer at high SZA values.

In all 3D MC runs identical upstream parameters were used to see the effect of the size of the object. The 3D MC model used periodic boundary conditions and the grid size was 0.125 times the radius of the object. The time steps in the 3D MC Moon, Phobos and RQ36 runs were 20s, 20s and 1s, respectively.

\subsubsection{Asteroid RQ36}

The first analysed object is the asteroid RQ36. Figure 6 shows the simulated normalized 3D dust densities when the roles of the initial velocity and the mass of a dust particle as well as the dust emission site were studied. In all cases in Fig. 6 the surface potential was assumed to be zero to study later its role. The charge of the dust particle was +350 e. The four runs, their basic input parameters and the main characteristic features of the solution are the following:

\section{Run RQ36-1 (Fig. 6a)}

No surface charging. Dust particles are emitted homogeneously through the entire surface of an object mimicking the situation when the asteroid is within large homogeneous and isotropic micrometeoroid cloud. The mass and the charge of a dust particle were $3.7 \times 10^{9}$ amu and +350 e, respectively. The initial bulk velocity in the Maxwellian velocity distribution function of the dust particles was zero but they had a non-zero temperature of $300 \mathrm{~K}$ which corresponds to the thermal speed of about $0.03 \mathrm{~m} / \mathrm{s}\left(=\sqrt{k_{B} T / m_{d u s t}}\right.$, where $k_{B}$ is Boltzmann's constant). Dust particles are bound to the surface and do not escape from the asteroid where the escape velocity is $0.2 \mathrm{~m} / \mathrm{s}$.

\section{Run RQ36-2 (Fig. 6b)}

In this case, the dust particles are injected from a single source point on the surface, mimicking the localised micrometeorite impact or emission caused by a human/robotic activity on the surface. The particles are injected from the source point located at $(x, y, z)=(\mathrm{R}, \mathrm{R}, \mathrm{R}) / \sqrt{ } 3$, where $R$ is the radius of the object, with initial velocity of $0.2 \mathrm{~m} / \mathrm{s}$, i.e. equal to the escape 
velocity. The dust particles can at this high velocity leave the object, while in the Run RQ36-1 the dust particles were all gravitationally bound to the asteroid.

\section{Run RQ36-3 (Fig. 6c)}

Simulation parameters for this run are identical to those in the $R u n R Q 36-2$, except that the mass of the dust particle was $3.7 \times 10^{8}$ amu, i.e., 10 times smaller than in the runs RQ36-1 and RQ36-2. The smaller mass results in a more extended dust cloud around the asteroid.

\section{Run RQ36-4 (Fig. 6d)}

In this run, the dust particles are released from a single point that is located in the $2 \mathrm{D} x y$-plane at $\mathrm{SZA}=45^{\circ}$ i.e. at $(x, y, z)=(\mathrm{R}, \mathrm{R}, 0) / \sqrt{ } 2$. The initial velocity for the dust particles in this simulation was increased to $0.4 \mathrm{~m} / \mathrm{s}$, i.e., to larger values than the escape velocity.

The effects of the surface charge in asteroid RQ36 was studied in Fig. 7. In this case, the inputs are similar to those in the two previous examples with the difference that the surface charge from the PIC model is included. The two new runs are the following.

\section{Run RQ36-5 (Fig. 7a)}

Simulation parameters for this run are similar to those in $R u n R Q^{36-1}$ except that the surface potential is now non-zero on the dayside. It can be seen that the surface potential charging has a considerable effect on dust motion near the asteroid, allowing dust particles to escape from the dayside in the analysed case with initial parameters. Note that dust particles cannot escape from the nightside because the surface potential in the model was zero there.

\section{Run RQ36-6 (Fig. 7b)}

In this example dust particles are released from a single source point on the surface which is on the $x y$-plane at $\mathrm{SZA}=45^{\circ}$. Simulation parameters are similar to those in Run RQ36-4, except that now the surface charging is included. Similarly, as in the Run RQ36-4, dust particles have an initial velocity prior to the surface charging. Therefore, the initial velocity of the particles is nearly 10 times larger than the escape velocity from the asteroid. In this case a jet-like stream of escaping dust particles is formed, as seen in Figure $7 \mathrm{~b}$.

Overall, when comparing Figs. 6 and 7, it is illustrated how the surface charging can affect the density distribution of the analysed dust particles. The detailed 3D density distribution depends, however, on the initial properties of the dust particles. Therefore, dust particles with different masses, charges and initial velocities can have very different 3D density profiles. The role of the initial parameters will be discussed later in detail in Section 2.3.4.

\subsubsection{Phobos moon}

The Martian moon Phobos is an example of an object with the size between the small asteroid RQ36 and the Moon. Figure 8 shows an example of simulation runs which are similar to those done for the asteroid RQ36.

\section{Run Phobos-1 (Fig. 8a)}

This run has similar inputs as the Run RQ36-1: Initial velocity, temperature, mass and the charge of dust particles are $0 \mathrm{~m} / \mathrm{s}, 300 \mathrm{~K}, 3.7 \times 10^{9}$ amu and +350 e, respectively. There is no surface charging and dust was emitted from the entire surface of Phobos. As in the asteroid Run RQ36- 
1 case dust particles do not have enough velocity to form an extended dust cloud far above the object.

\section{Run Phobos-2 (Fig. 8b)}

In this run, all the simulation parameters are similar to those in Run Phobos-1, but now the dust emission is from a single point source which is on the $x y$-plane at SZA $=45^{\circ}$. Note that the initial velocity of a dust particle $\left(U=12.1 \mathrm{~m} / \mathrm{s}, T=5 \times 10^{7} \mathrm{~K}\right)$ is larger than the escape velocity from Phobos $(\sim 11 \mathrm{~m} / \mathrm{s})$. Note also the formation of a dust plume around Phobos.

\section{Run Phobos-3 (Fig. 8c)}

The simulation parameters for this run are similar to those in Run Phobos-1 except that now the non-zero surface charging is included on the dayside. The surface potential is similar to the surface potential applied in $\mathrm{R} u n \mathrm{R} Q 36$. Note how the surface potential accelerates dust particles upward, and a clear 3D dust cloud is formed around Phobos on the dayside.

\section{Run Phobos-4 (Fig. 8d)}

This run had the same inputs as those in Run Phobos- 3 but now the mass of a dust grain was reduced ten times from $3.7 \times 10^{9}$ amu to $3.7 \times 10^{8}$ amu. As can be seen in Fig. 8d, the surface potential for these light dust particles has resulted in an even more extended 3D dust cloud.

\subsubsection{The Moon}

The Moon is larger and much heavier than Phobos and RQ36, thus the surface charging does not provide enough energy for the released dust particles to escape from the lunar gravitational field. Therefore, to see dust far above the surface, dust particles have been assumed to have an initial velocity of $3 \mathrm{~km} / \mathrm{s}$, i.e., larger than the escape velocity from the Moon $(\sim 2.5 \mathrm{~km} / \mathrm{s})$. The mass and the charge of

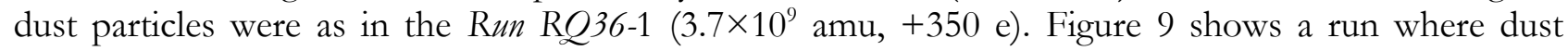
particles were emitted from a single point at $\mathrm{SZA}=0^{\circ}$. Such emission results in density enhancement on the dayside around the subsolar point.

\section{Phase space and the role of the Debye layer}

The PIC model has a special role in the DPEM modelling suite because it transfers information from the hybrid model to the $\mathrm{MC}$ model by linking the properties of the 3D plasma environment and the 3D dust environments. Connecting the hybrid model and the MC model was achieved via the surface potential. The effect of the surface potential on the dust densities has been discussed briefly already in Section 2.3.2, but it is useful to examine it quantitatively. This can be done by analysing Eq. (7) and writing it in the form

$$
\begin{aligned}
& v_{r}^{M C}(S Z A)=v_{r}^{\text {Maxwell } \sqrt{1+c_{\varphi}-c_{g}}} \\
& c_{\varphi} \equiv \frac{E_{\varphi}^{\text {pot }}}{E_{r}^{\text {kin }}}=\frac{q_{\text {dust }} \varphi_{\text {surf }}^{\text {PIC }}(S Z A)}{1 / 2 m_{\text {dust }} v_{r}^{\text {Maxwell }}{ }^{2}} \\
& c_{g} \equiv \frac{E_{g}^{\text {pot }}}{E_{r}^{\text {kin }}}=\frac{m_{\text {dust }}\left(U(h)_{g}-U(0)_{g}\right)}{1 / 2 m_{\text {dust }} v_{r}^{\text {Maxwell }^{2}}}
\end{aligned}
$$


The last term in Eq. (8a) on the right hand side, $c_{g}$, does not include effects of the Debye layer but only non-electromagnetic effects. Effects of the Debye layer are in the term $c \varphi$, which is the ratio between the electric potential of a dust particle within the Debye layer and the kinetic energy of a dust particle on the surface. If $c \varphi$ is larger than 1 , the radial velocity is increased, and if $c \varphi$ is smaller than one the radial velocity is decreased by the Debye layer. Let us now consider the case when $c_{\varphi}>>c_{g}$ which can take place when the Debye layer is very narrow, the dust particle is light, the surface potential is strong or when the dust particle is strongly charged. In this case the initial radial velocity is changed by a factor of $\sqrt{1+c_{\varphi}}$ when it exits from the Debye sheath.

Table 1 gives the summary of the evaluated 2D and 3D MC runs and of the input parameters for the dust particles. It also shows the corresponding values for $c_{\varphi}$ in the cases when the bulk velocity was non-zero.

The $\left(v_{0}, m / q\right)$-phase space plot in Fig. 10 shows how $c_{\varphi}$ depends on the $(m / q)$ ratio of a positively charged dust particle with initial velocity $v_{o}$ at the SZA $=0^{\circ}$ case where the surface charge in the analysed PIC simulation was about $+3 \mathrm{~V}$ on the surface (c.f. Fig. 4). Note that Figure 10 divides the $\left(v_{0}\right.$, $\mathrm{m} / \mathrm{q}$ )-phase space into two regions. In the region $c_{\varphi}<0.1$ the contribution of the surface potential to the initial velocity is small because the acceleration is less than five percent $(\sqrt{1+0.1}=1.0488)$. In this parameter range the $3 \mathrm{D}$ density profile in the $\mathrm{MC}$ simulation depends on the initial velocity, and the PIC simulation results are not needed in the 3D MC simulation. In the region $c_{\varphi}>10$, instead, the role of the Debye layer is important because the radial velocity of a dust particle which has passed the layer is over three times the initial radial velocity $(\sqrt{1+10}=3.3166)$. In this range the PIC simulation forms a "link" in the DPEM model between the 3D hybrid model and the 3D MC models.

Figure 10 also illustrates how the six $\left(v_{0}, \mathrm{~m} / \mathrm{q}\right)$ cases analysed in this paper differ from each other in terms of $c \varphi$. Initial dust parameters on the Phobos and asteroid RQ36 cases are chosen in the region where the surface charging is important while the dust charging is not important in the analysed Moon case. The local 2D MC simulation was an "intermediate" case between the Phobos/asteroid RQ36 and the Moon cases. The dust cases analysed in this study provide therefore examples of cases where the role of the surface charging varies. It should also be noted than in the MC model the surface charging was assumed to be zero on the nightside. According to Fig. 10 this can be interpreted as mimicking a situation where the phase space point $\left(v_{0}, m / q\right)$ was in the $c_{\varphi}<0.01$ region.

\subsection{Charge and initial velocity of a dust particle}

One highly critical yet unknown initial condition, which is needed to estimate properties of dust, in addition to the initial velocity of a dust particle, is the electric charge. It is illustrative to estimate what the charge of a dust particle on the object surface would be in the analysed surface electric field cases.

One way to estimate the charge of the surface and the dust on it is to assume that the charge is homogeneously distributed on a planar surface, that is, to assume there is a constant surface charge density $\sigma\left[\mathrm{C} / \mathrm{m}^{2}\right]$ on the surface of all material particles connected to the surface. If the electric field on the surface is $E_{\text {surf }}$, then $\sigma$ is equal to $2 \epsilon_{\mathrm{o}} E_{\text {sur }}$ according to Gauss' law. In the analysed SZA $=0^{\circ}$ case $E_{\text {suff }} \sim+3 \mathrm{~V} / \mathrm{m}$ and, therefore, $\sigma \sim 5.31 \times 10^{-11} \mathrm{C} / \mathrm{m}^{2} \sim 3.3 \times 10^{-4} \mathrm{e} / \mu \mathrm{m}^{2}$. This shows that only about less than one of every 10 thousand micron size dust particle has an electron while the other dust particles are non-charged.

If we then assume that a spherical dust particle of the radius $r_{\text {dust }}$ and the surface area of $A_{\text {dust }}$ has the same surface charge density as the surface, the charge of the dust particle on the surface is 


$$
\mathrm{q}_{\text {dust }}[\mathrm{e}]=\mathrm{A}_{\text {dust }} \sigma=4 \pi \mathrm{r}_{\text {dust }}^{2} \sigma \sim 0.0042 \mathrm{r}_{\text {dust }}^{2}[\mu \mathrm{m}]
$$

Here the electric charge is given in the unit of the elementary charge $e$ and the radius is given in $\mu \mathrm{m}$. As Eq. 9 indicates, the charge of a micron size dust particle is very small in the analysed cases. For example, the radius of a dust particle has to be about $15 \mu \mathrm{m}$ for the dust particle to carry the charge of an electron, and about $150 \mu \mathrm{m}$ to have a charge of 100 electrons, which is in the order of the charge of a dust particle analysed in the paper. Such a particle would be very large and, consequently, very heavy. For example, if we assume a spherical dust particle with a mass density, $\rho_{\text {dust }}^{\text {mass }}$, of $3 \mathrm{~g} \mathrm{~cm}^{-3}$ the mass of a 1e particle would be $\sim 3 \times 10^{16}$ amu and for a 100e particle would be $\sim 3 \times 10^{19}$. This means that the $m_{\text {dust }} / q_{\text {dust }}$ ratio of a $1 \mathrm{e}$ and a $100 \mathrm{e}$ dust particle would be $\sim 3 \times 10^{16} \mathrm{amu} / \mathrm{e}$ and $\sim 3 \times 10^{17} \mathrm{amu} / \mathrm{e}$, respectively. These high mass values are outside the range in Fig. 10 and belong to the region where the Debye layer is not important.

This analysis suggests that if the surface is made of equal size spherical charged particles with size one micron or less, most of the dust particles would have zero charge. A more accurate estimation should take into account non-spherical shaped dust particles. A sphere is a geometrical shape where the surface area/volume ratio is in its minimum. Therefore, the mass of a non-spherical particle associated with the $A_{\text {dust }}$ is smaller than for a spherical particle. It is, however, unlikely that a non-spherical "fluffy" shape of a particle could decrease the mass per charge ratio many orders of magnitude.

Therefore, some other more effective dust charging mechanism than photoemission by EUV is needed to result in dust charges analysed in this paper if we assume a homogeneously distributed electric charge on a planar surface. Other surface charging sources could be X-rays [Manka, 1973] or micrometeoroid impact ionization (e.g. Collette et al., 2014). Secondary electrons also result in surface charging and their role has been studied widely, for example, when spacecraft charging has been analysed (e.g. Pavlŭ et al. 2014). Laboratory measurements have shown that dust particles are mobilized and transported on the surface near regions of differing secondary electron yields due to either their characteristic compositions or surface roughness, and that a few $\mathrm{kV} / \mathrm{m}$ electric field can be formed near the surface [Wang et al., 2010]. However, it is not clear how well the laboratory measurements can mimic real situations on the lunar surface and the complex surface/structure of the dust particles. Moreover, the question of how large the charge of a dust particle can be and what mechanism could lift dust particles from the surface is unknown.

The phase space shown in Figure 10 illustrates the parameter range where the surface charging can have global effects on dust densities, i.e., when surface charging has substantial effects on the velocity of a dust particle after it has escaped from the Debye layer. However, it is also informative to analyse local effects, that is, a situation when the initial velocity of a dust particle is large enough to affect its motion within the Debye layer. It is interesting to consider how small a dust particle on a surface can be so that after emission of the minimum amount of electric charge, i.e., one photoelectron, it would have such a small $\mathrm{m} / \mathrm{q}$ ratio that the surface potential would be important. If we assume again that the mass density of a spherical dust particle is $3 \mathrm{~g} \mathrm{~cm}^{-3}$, the radius of a particle which has emitted one photoelectron and which $\mathrm{m} / \mathrm{q}$ ratio is $1 \times 10^{11} \mathrm{amu} / \mathrm{e}, 1 \times 10^{10} \mathrm{amu} / \mathrm{e} 1 \times 10^{9} \mathrm{amu} / \mathrm{e}$ and $1 \times 10^{8} \mathrm{amu} / \mathrm{e}$ is $\sim 0.2 \mu \mathrm{m}, \sim 0.1 \mu \mathrm{m}, \sim 0.05 \mu \mathrm{m}$, and $\sim 0.02 \mu \mathrm{m}$, respectively. According to Fig. 10 those particles are close to the $\mathrm{c}_{\varphi}=1$ line if their initial radial velocity is of the order of $\sim 0.06 \mathrm{~km} / \mathrm{s}, \sim 0.3 \mathrm{~km} / \mathrm{s}, \sim 0.8$ $\mathrm{km} / \mathrm{s}, \sim 3 \mathrm{~km} / \mathrm{s}$, respectively. This implies that for a dust particle of radius, say, $\sim 0.02 \mu \mathrm{m}$ the initial velocity becomes locally important if it is of the order of $1 \mathrm{~km} / \mathrm{s}$, as demonstrated in $1 D$ PIC simulations which showed that the maximum velocity of a levitating $0.02 \mu \mathrm{m}$ dust particle is only few $\mathrm{m} / \mathrm{s}$ (see Poppe and Horànyi, 2010, Fig. 11). However, it is not known how a dust particle could have an initial velocity of the order of $\mathrm{km} / \mathrm{s}$. 


\section{Discussion}

This study describes the new unified DPEM modelling suite of three integrated kinetic models that enables local and global analysis of dust and plasma near an airless planetary object. The 3D hybrid model uses a hybrid modelling package built on the publicly available FLASH code (http://flash.uchicago.edu/site/flashcode/), which has already been used to study the Moon-solar wind interaction [Holmström et al., 2012]. The 3D MC model was also built around the FLASH code, and it uses a new dust software package developed for the DPEM model. The PIC model is a new code utilising the HYB software platform, which included initially a hybrid model (e.g. Kallio, 2005, and references therein), a 3D electromagnetic model, and then a 1D electrostatic model. Recently, the developed 2D PIC model has been extended to model also a non-planar surface [Dyadechkin et al., 2015].

The DPEM model provides a tool to analyse many aspects associated with the plasma and dust near the surface of an airless body, such as 1) the properties of plasma near the surface based on a 3D kinetic model (hybrid model), 2) to study electric potential and the dust density profiles within and near the surface (PIC model) and, finally, 3) to obtain 3D dust density profiles. The analyses imply that properties of the lifted dust strongly affect the dust densities locally and globally depending on the mass, charge and initial velocity of the dust.

Although the DPEM model links three individual models which are dedicated to analysing different plasma processes and plasma phenomena, the modelling suite can be regarded as the first step towards a real unified model for studying the dust environment of airless bodies. The one direction connection between the models Hybrid $\rightarrow$ PIC $\rightarrow$ MC (c.f. Fig. 1) manifests the challenge of the physical processes in a wide range of space and time scales. The fastest processes in the modelling suite are the electron plasma frequency $\left(\sim 9 \sqrt{n}_{n_{e}}\left[\mathrm{~cm}^{-3}\right] \mathrm{kHz}\right)$ and the smallest space scale is the Debye length $(\sim 69 \sqrt{ }$ $\left.T_{e}[\mathrm{~K}] / n_{e}\left[\mathrm{~m}^{-3}\right] \mathrm{m}\right)$ that are considered in the PIC model. Therefore, for example, in the PIC model the time step in the simulation, the running time, and the grid size were $5 \times 10^{-8} \mathrm{~s}, 2 \mathrm{~ms}$ and $0.25 \mathrm{~m}$, respectively. These values were much smaller than those used in the MC model which considered the slowly moving dust particles in a 3D space. For example, in Figs. 6-9 the running time of the simulation was thousands of seconds.

Because of the highly different time and space scales in the three DPEM models, dust particles did not affect the properties of plasma in the PIC or hybrid model. In a completely unified model, instead, the link in a dusty-plasma model should be two way (Hybrid $\leftrightarrow$ PIC $\leftrightarrow$ MC). For example, dust particles can be a source of electrons, the so called dust electrons (e.g. Stubbs et al., 2011), which in turn affect the electron density and electron distribution function. A self-consistent model should include this effect into the PIC model. Moreover, the charge of a dust particle above the surface varies in time and it depends on the nearby plasma (see e.g. Nitter et al., 1998) while the charge of a dust particle was kept constant in the 2D PIC simulation (c.f. Fig. 5) and in the 3D MC simulations (c.f. Figs. 6-9) for simplicity. It is however a very challenging task to model the whole system fully self-consistently with present computational resources.

The nightside region was also modelled in a simpler manner by using a zero surface potential. This approach was chosen because the hybrid model does not give accurate plasma parameters near the surface by or at midnight. It can be envisioned that a more realistic 3D dust distribution could be obtained when the PIC model uses, for example, observations or some other models. In addition, 2D landscapes can affect the electric potential and they should be included into the PIC model (see e.g. Zimmerman et al., 2011). Lunar magnetic anomalies also affect the properties of the precipitating solar wind protons and the electric potential near the surface (see, e.g. hybrid modelling by Jarvinen et al., 2014). In the future, such magnetic field regions could be simulated with the DPEM model by 
combining the 3D hybrid model which simulates magnetic anomaly globally and a local PIC simulation which gives electric potential within the Debye layer.

It should also be mentioned, that although the hybrid model in the DPEM suite enables to derive detailed knowledge of the properties of the solar wind protons near the terminator region and then used in the PIC simulation, in the analysed cases the detailed 3D velocity distribution information was not used in the PIC model. The reasons for this choice is based on the relatively small level of disturbance observed (c.f. Fig 3). However, if the goal of the study would have been to analyse local property of the dust near the terminator region, for example, near the lunar polar regions, then the detailed velocity distribution function obtained from the hybrid model would have been highly important. It would also be interesting to test how the properties of the plasma differ near the terminator region at different solar wind conditions and, for example, when the Moon is in the Earth's magnetosphere or when Phobos is in the induced Martian magnetosphere. Moreover, the hybrid model can also simulate cases when the solar wind plasma is non-Maxwellian. Such studies are, however, beyond the scope of the present work.

To interpret the results obtained in this study, it is important to bear in mind that the DPEM modelling suite does not model the initial dust particle injection from the surface of the planetary body. In other words, the DPEM model does not include information of how many dust particles are emitted at various places in the ( $\left.\mathrm{v}_{\mathrm{o}}, \mathrm{m} / \mathrm{q}\right)$-phase space shown in Fig. 10. Therefore, the DPEM modelling suite cannot provide absolute dust density profiles without inclusion of an additional dust lifting modelling, based on the laboratory measurements of hypervelocity impacts, for example (see e.g. Frisch, 1992; Eichhorn and Grün, 1993; Lange and Ahrens, 1987), or dust lifting models based on the first physical principles of the forces affecting dust particles on the surface of an airless body (see e.g. Hartzell and Scheeres, 2011; Hartzell, 2012). For example, as can be seen in Fig. 10, the initial parameters of dust particles injected from the surface of RQ36 (Fig. 6-7) and Phobos (Fig. 8) were chosen in the phase space so that the surface electric potential has a significant contribution to the density of dust.

Modelling is also complicated due to the fact that the shape and surface area of the dust particles are highly irregular and relatively large, respectively (e.g. Liu et al., 2008 and Liu and Taylor, 2011). To be able to include these input parameters would be crucial to obtain a realistic 3D dust density profile. Therefore, more theoretical studies and laboratory measurements are called for to characterise more precisely the properties of lifted dust particles and the potential variability due to different bulk chemical composition. Moreover, different lunar missions and different observations have provided contradictory results about the properties of dust around the Moon (see e.g. Feldman et al., 2014) and, therefore, more dedicated remote sensing and in situ dust observations are needed. This will help in further understanding the interaction of the charged particles and the potential "dangling" surface bonds (free radicals) with the exterior and interior of the human body (e.g. Fubini and Hubbard, 2003; and Hurowitz et al., 2007) and technical instrumentation used on the lunar surface. This will enable a better understanding of the potential toxicity of the lifted dust particles and respective hazards to human health. Moreover, the improvements in models can also help to better protect scientific and technical instrumentation used on the lunar surface.

\section{Summary}

A new tool called the DPEM modelling suite has been developed to study dust environments of airless objects. This unified kinetic model was used to study three different planetary object sizes (the Moon, the Martian moon Phobos and the asteroid RQ36). The phase space analysis revealed regions in the 
initial velocity - the mass-to-charge ratio phase space where the density of the dust particles was affected strongly by the electric field within the Debye layer. The study also showed how the 3D density distribution of such dust particles is affected by the Debye layer. Overall, the initial results presented in this paper suggested that when a realistic dust emission model is available, the unified DPEM modelling suite provides a powerful tool to study the dust environments of airless bodies such as moons, Mercury, asteroids and non-active comets far from the Sun.

\section{Acknowledgements}

The authors acknowledge the ESA GSP program project DPEM, Dusty plasma environments: nearsurface characterization and modelling (ESA ITT, AO-1-6696/11/NL/CO) for the financial support for the work. The authors thank Fabrice Cipriani/ESTEC/ESA for the discussions about dusty plasma. Authors would like to acknowledge Dr. D. Kent Ross (NASA-JSC) for proofreading the manuscript and for useful comments and remarks. EK thanks Dr. Cyril Simon Wedlund, Aalto University, for useful suggestions about the manuscript. Figs. 2, 6, 7, 8 and 9 are made by the VisIt visualization tool.

\section{References}

Cain J. R., Lunar dust: the hazard and astronaut exposure risks, 2010, Earth, Moon, Planets 107, 107125.

Collette, A., E. Grün, D. Malaspina, Z. Sternovsky, 2014, Micrometeoroid Impact-Charge Yield for Common Spacecraft Materials, J. Geophys. Res., doi: 10.1002/2014JA020042.

Dyadechkin, S., E. Kallio, and P. Wurz, 2015, New fully kinetic model for the study of electric potential, plasma, and dust above lunar landscapes, J. Geophys. Res. Space Physics, 120, doi:10.1002/2014JA020511.

Eichhorn, K. and G. Eberhar, 1993, High-velocity impacts of dust particles in low-temperature water ice, Planetary and Space Science, Volume 41, Issue 6, pp. 429-433, doi:10.1016/0032-0633(93)90002-J.

Farrell, W.M., Stubbs, T.J.,Vondrak,R.R., Delory, G.T., Halekas, J.S., 2007, Complex electric fields near the lunar terminator: the near-surface wake and accelerated dust, Geophysical Research Letters 34,L14201, http://dx.doi.org/10.1029/ 2007GL029312.

Feldman P. D., D. A. Glenar, T. J. Stubbs, K. D. Retherford. R. Gladstone, P. F. Miles, T. K. Greathouse, D. E. Kaufmann, J. Wm. Parker, S. A. Stern, 2014, Upper limits for a lunar dust exosphere from far-ultraviolet spectroscopy by LRO/LAMP, Icarus 233, pp. 106-113.

Frisch, W., 1992, Hypervelocity impact experiments with water ice targets in Hypervelocity Impacts in Space, Edited by McDonnell. J. A. M., pp. 7-14. Unit for Space Sciences, University of Kent.

Fubini B. and Hubbard A., 2003, Serial Review: Role of Reactive Oxygen and Nitrogen Species (ROS/RNS) in Lung Injury and Diseases. Free Radical Biology \& Medicine 34, 1507-1516.Holmström, M., Fatemi, S., Futaana,Y., Nilsson, H., 2012, The interaction between the Moon and the solar wind, Earth, Planets and Space64, 237-245, http://dx.doi.org/10.5047/eps.2011.06.040. 
Hartzell, C. M. and D. J. Scheeres, 2011, The role of cohesive forces in particle launching on the Moon and asteroids, Planetary and Space Science 59, pp. 1758-1768.

Hartzell, C., 2012, The dynamics of near-surface dust on airless bodies, Ph.D. Thesis, University of Colorado.

Hurowitz J.A., Tosca N.J., McLennan S.M. and Schoonen M.A.A., 2007, Production of hydrogen peroxide in Martian and lunar soils. Earth and Planetary Science Letters 255, pp. 41-52.

Jarvinen, R., M. Alho, E. Kallio, P. Wurz, S. Barabash, and Y. Futaana, 2014, On vertical electric fields at lunar magnetic anomalies, Geophys. Res. Lett., 41, 2243-2249, doi:10.1002/2014GL059788,

Kallio, E., J. G. Luhmann, and S. Barabash, 1997, Charge exchange near Mars: The solar wind absorption and neutral atom production, J. Geophys. Res., 102, 22, 183-22,197.

Kallio, E., Formation of the lunar wake in quasi-neutral hybrid model, 2005, Geophysical Research Letters, 32, L06107,http://dx.doi.org/10.1029/2004GL021989.

Kallio, E., R. Jarvinen, S. Dyadechkin, P. Wurz, S. Barabash, F. Alvarez, V. Fernandes, Y. Futaana, J. Heilimo, C. Lue, J. Makela, N. Porjo, W. Schmidt and T. Siili, 2012, Kinetic simulations of finite gyroradius effects in the lunar plasma environment on global, meso, and microscales, Planetary and Space Science, http://dx.doi.org/10.1016/j.pss.2012.09.012.

Kallio, E. and G. Facskó, Properties of plasma near the Moon in the magnetotail, Planetary and Space Science, doi:10.1016/j.pss.2014.11.007, 2015.

Dyadechkin, S., E. Kallio, P. Wurz, 2015, New fully kinetic model for the study of electric potential, plasma and dust above lunar landscapes, J. Geophys. Res., DOI: 10.1002/2014JA020511,.

Jordan, A. P., T. J. Stubbs, J. K. Wilson, N. A. Schwadron, and H. E. Spence, 2015, Dielectric breakdown weathering of the Moon's polar regolith, J. Geophys. Res. Planets, 120, 210-225, doi:10.1002/2014JE004710.

Lange, M. A. and T. J. Ahrens, 1987, Impact experiments in low-temperature ice, Icarus, Volume 69, Issue 3, pp. 506-518, doi:10.1016/0019-1035(87)90020-0

Linnarsson, D., J. Carpenter, B. Fubini, P. Gerde, L. L. Karlsson, D. J. Loftus, G. K. Prisk, U. Staufer, E. M. Tranfield and W. van Westrenen, 2012, Toxicity of lunar dust, Planetary and Space Science 74, $57-71$.

Liu Y. and Taylor T. A., 2011, Characterization of lunar dust and a synopsis of available lunar simulants. Plan. Space Sci. 59, 1769-1783.

Liu Y., Park J. S., Schnare D., Hill E. and Taylor L. A., 2008, Characterization of lunar dust for toxicological studies: Part 2-morphology and physical characteristics. J. Aerosp. Eng. 21, 272-279.

Manka, R.H. Plasma and potential at the lunar surface, 1973, in: Grard, R.J.L. (Ed.), Photon and Particle Interactions with Surfaces in Space. D. Reidel Publishing Co., Dordrecht, Holland, pp. 347361. 
Murphy, D.L., Vondrak, R.R., 1993, Effects of levitated dust on astronomical observations from the lunar surface. Proc. Lunar Planet. Sci. Conf. 24th, 1033-1034.

Nitter, T., Havnes, O., Melandso, F., 1998, Levitation and dynamics of charged dust in the photoelectron sheath above surface in space, J. Geophys. Res., 103(A4), 6605-6620, http://dx.doi.org/10.1029/97JA03523.

Pavlŭ, J., M. Beránek, J. Vaverka, J. Šafránková, Z. Nemeććek, and I. Richterová, 2014, Secondary electron emission from Martian soil simulant, J. Geophys. Res. Planets, 119, 199-209, doi:10.1002/2013JE004522.

Poppe, A., and M. Horànyi, 2010, Simulations of the photoelectron sheath and dust levitation on the lunar surface, J. Geophys. Res., 115, A08106, doi:10.1029/2010JA015286.

Stubbs T. J., Vondrak R. R. and Farrell W. M., 2006, A dynamic fountain model for lunar dust. Adv. Space Res. 37, pp. 59-66, 65.

Stubbs, T.J., Glenar, D.A., Farrell, W.M., Vondrak, R.R., Collier, M.R., Halekas, J.S., Delory, G.T., 2011, On the role of dust in the lunar ionosphere, Planetary and Space Science, http://dx.doi.org/10.1016/j.pss.2011.05.011.

Wang, X., Horànyi, M., Robertson, S., 2010, Investigation of dust transport on the lunar surface in a laboratory plasma with an electron beam. J. Geophys. Res. 115, A11102, pp. 1-6.

Willis, R. F., M. Anderegg, B. Feuerbacher, and B. Fitton, 1973, Photoemission and secondary electron emission from lunar surface material, in Photon and Particle Interactions With Surfaces in Space, edited by R. J. L. Grard, pp. 369-387, Springer, New York.

Zimmerman, M. I., W. M. Farrell, T. J. Stubbs, J. S. Halekas, and T. L. Jackson, 2011, Solar wind access to lunar polar craters: Feedback between surface charging and plasma expansion, Geophys. Res. Lett., 38, L19202, doi:10.1029/2011GL048880. 


\section{Figure Captions}

Figure 1. Overview of the three models in the DPEM modelling suite: [1] a 3D Hybrid model, [2] a 2D electrostatic full kinetic Particle-In-Cell (PIC) model, and [3] a 3D Monte Carlo (MC) model. All three models have also their own 3D Maxwellian Particle Generator, MPG, to inject particles into models. Models are connected to each other by a one-way relationship: The PIC model uses the impacting $\mathrm{H}^{+}$ ions derived by the hybrid model while the MC model uses the surface potential calculated by the PIC model. Input parameters for the models are the solar wind $(\mathrm{SW})$, the radius of the object $\left(\mathrm{R}_{o b j}\right)$, the gravitational force $(g)$ and the interplanetary magnetic field $\left(B_{s w}\right)$. The physical output dust parameters are $1 \mathrm{D}, 2 \mathrm{D}$ and $3 \mathrm{D}$ density profiles.

Figure 2. An example of a run made by the DPEM hybrid model. The colour gives the magnitude of the magnetic field normalized by the undisturbed magnetic field on two planes: (a) on the $z=0$ plane and (b) on the $y=0$ plane. The solar wind flows in the figure from right to left and the Moon is assumed to be in the undisturbed solar wind.

Figure 3. Macroscopic properties of the $\mathrm{H}^{+}$protons precipitating on the surface of the Moon (blue lines), Phobos (pink lines), and asteroid RQ36 (red lines). In panels b) and c) the solid, dash-dot and the dotted lines show the $\mathrm{x}, \mathrm{y}$ and $\mathrm{z}$ components, respectively. The bottom panel shows the number of precipitated $\mathrm{H}^{+}$ions on the surface into $10^{\circ}$ intervals of the SZA. The vertical dotted line at SZA $=90^{\circ}$ shows the position of the terminator plane which separated the dayside and nightside hemispheres. Note that in panel d) the maximum number of collected particles is obtained in the region $40^{\circ}<\mathrm{SZA}$ $<50^{\circ}$ because the area of that ring on the lunar surface perpendicular to the flow of the solar wind is larger than the perpendicular area in other $10^{\circ}$ intervals. Note also that the statistics of the derived macroscopic parameters is best in the SZA regions where number of the collected macroparticles is largest.

Figure 4. 1D profiles of the electric potential derived from a 1D electrostatic PIC simulation for six SZA values.

Figure 5. Analysis of the role of the surface charging to the density of dust within the Debye layer. The curves show the normalized density of dust particles at six SZA cases for the (a) "cold" dust run (the Run PIC-1) and (b) the "hot" dust run (the Run PIC-2) when the thermal velocity ( $\mathrm{V}_{\text {th }}$ ) was small, large compared with the bulk velocity, respectively. The densities are calculated with the $2 \mathrm{D} \mathrm{MC}$ model by using the electric potential given in Fig. 4. In (a) every six cases are normalized so that the maximum density is one. In (b) densities are normalized with the maximum density of dust in the SZA $=0^{\circ}$ case.

Figure 6. Normalized dust densities around asteroid RQ36 on the (left columns) $x y$-plane and (right columns) on the $x$ \%-plane when the surface potential is not included. In the first run (RQ36-1) dust was emitted from the full surface while in the rest three runs (RQ36-2, RQ36-3, RQ36-4) dust was emitted from a single point. Legends of the panels show the input parameters: The initial velocity $\left(v_{d}\right)$ or temperature $\left(T_{d}\right)$ of a dust particle and its mass $\left(m_{d}\right)$. In all cases the charge of the dust particle was +350 e. The densities in panels a), b), c) and d) are snapshots at $6000 \mathrm{~s}, 7500 \mathrm{~s}, 7500 \mathrm{~s}$ and $7500 \mathrm{~s}$ after the continuous emission was started, respectively. In the plots the Sun is on the right.

Figure 7. Normalized dust densities around asteroid RQ36 on the (left columns) $x y$-plane and (right columns) on the $x$-plane when the surface potential is included. The run on the top (RQ36-5)/bottom (RQ36-O) is otherwise similar than the Run RQ36-1/ RQ36-4 shown in Fig. 6. The densities in panels a) 
and b), are snapshots at $6000 \mathrm{~s}$ and $7500 \mathrm{~s}$ after the continuous emission was started, respectively. In the plots the Sun is on the right.

Figure 8. Normalized dust densities around Martian moon Phobos on the (left columns) $x y$-plane and on the (right columns) $\times$ ₹-plane at four MC model runs. In panels a) and b) there is no surface charging but in panels c) and d) the surface charging is added. Dust was emitted from the full surface in panels a) and c) and d) while the panel b) shows the point source situation. The densities in panels a), b), c) and d) are snapshots at $10000 \mathrm{~s}, 7500 \mathrm{~s}, 10000 \mathrm{~s}$ and $40000 \mathrm{~s}$ after the continuous emission was started, respectively. In the plots the Sun is on the right.

Figure 9. Normalized dust densities around the Moon on the $x y$-plane derived from the 3D MC simulation. Dust particles were emitted at the subsolar point $\left(\mathrm{SZA}=0^{\mathrm{O}}\right)$. The densities are derived after $5000 \mathrm{~s}(\sim 1.4 \mathrm{~h})$ continuous dust emission.

Figure 10 .

The $\left(v_{0}, \mathrm{~m} / \mathrm{q}\right)$-phase space where the analysed dust simulations are marked in the $2 \mathrm{D}$ phase space according to the initial velocity $\left(v_{0}\right)$ and the initial mass per charge $(\mathrm{m} / \mathrm{q})$ ratio of the dust particles. The ratio $\left(c_{\varphi}\right)$ of the electric potential energy of a positive dust particle to the vertical kinetic energy at different $(\mathrm{m} / q)$ and initial radial velocity $\left(v_{0}\right)$. The three lines present $c_{\varphi}=0.1,1$, and 10 cases. The marks represent six initial $\left(v_{0}, m / q\right)$ parameter pairs used in the local 2D simulation with the Debye layer (Runs PIC-1 and PIC-2) and in the 3D MC simulations for the Phobos moon, the asteroid RQ36, for the Moon simulation.

\section{Tables}

Table 1. Summary of the analysed MC runs and the input parameters used for the dust particles. The dust is emitted from the surface with a 3D Maxwellian velocity distribution function with the radial bulk velocity $\left(U_{\text {dust }}\right)$ and temperature $\left(T_{\text {dust }}\right)$ or the thermal speed $\left(U_{\text {dberm }}^{\text {dust }}\right)$. The second last column on the left is a parameter $c_{\varphi}$ which represents the role of the surface charge in Eq. $8 \mathrm{~b}$. The charge and the mass of the dust particle is given in elementary charge ( $=$ the charge of an electron) and atomic mass unit, amu, ( $\sim$ the mass of a proton), respectively. The velocity used to calculate $c_{\varphi}$ in the PIC-2 run was taken to be $12 \mathrm{~m} / \mathrm{s}\left(=U_{\text {dust }}+U^{\text {therm }}\right.$ dust $)$ for simplicity. Values $c \varphi$ are derived for the surface potential $+3 \mathrm{~V}$ which is the case near the subsolar point in the analysed input parameter cases. 
Fig. 1.

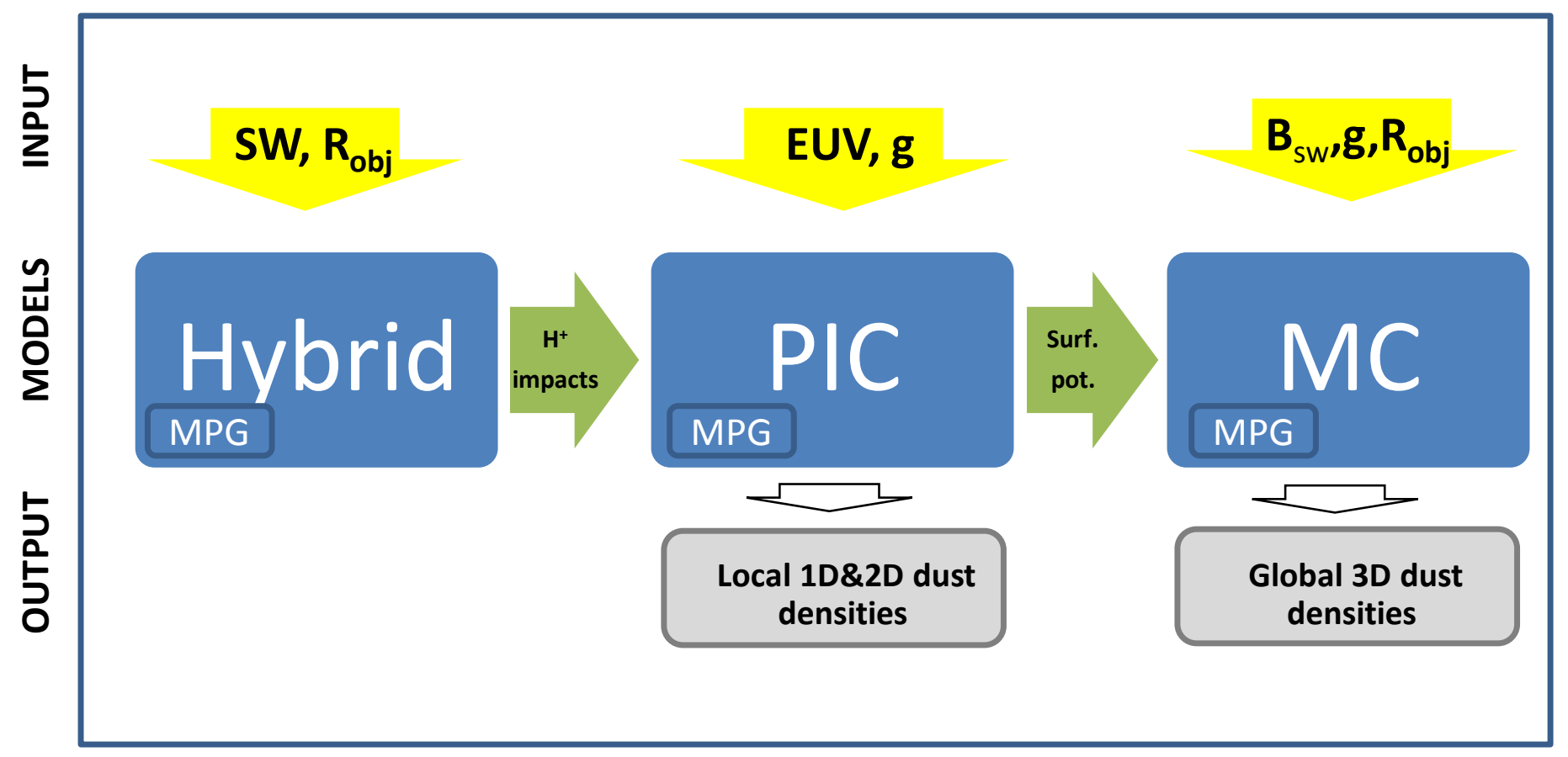


Fig. 2.
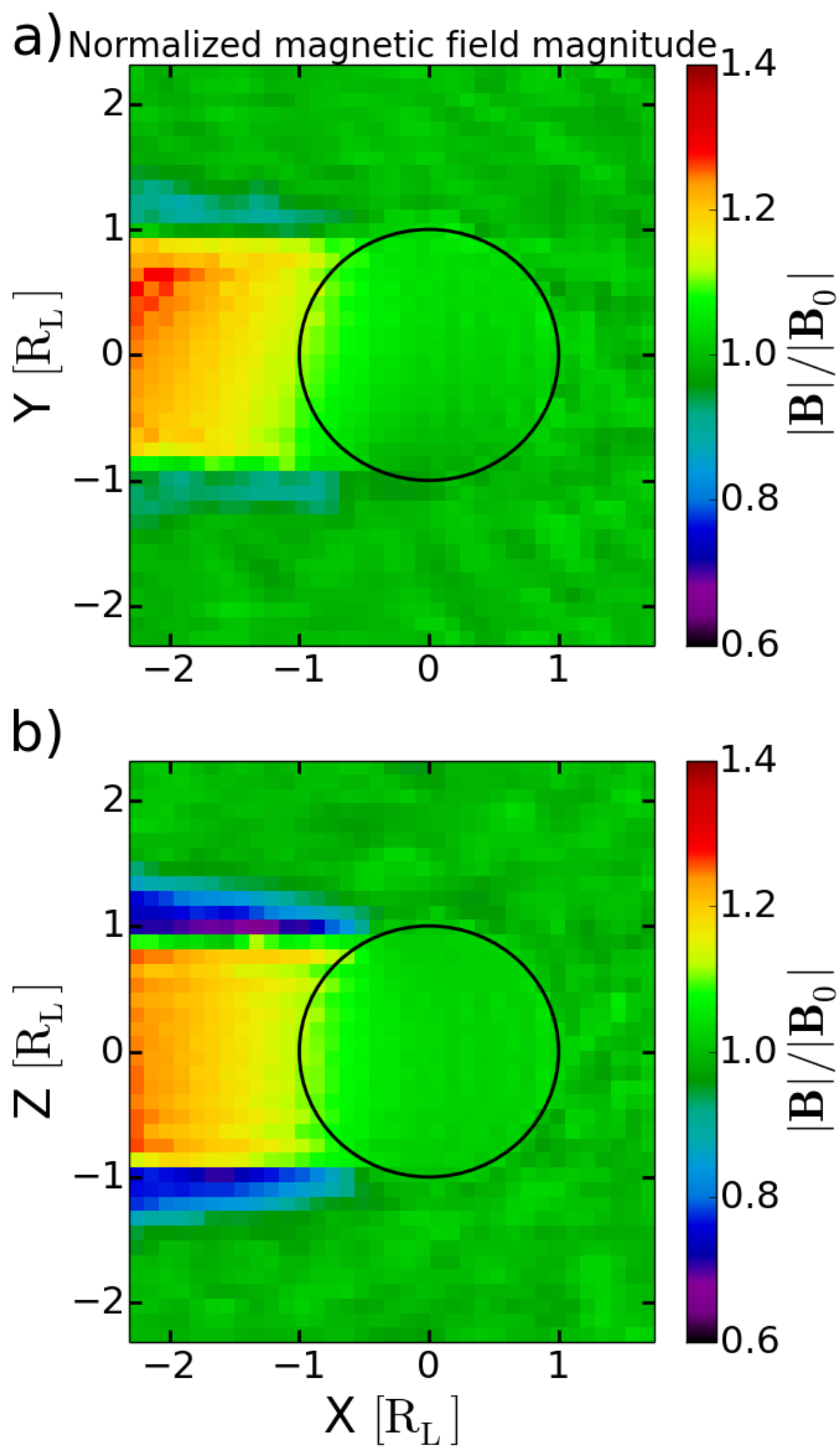

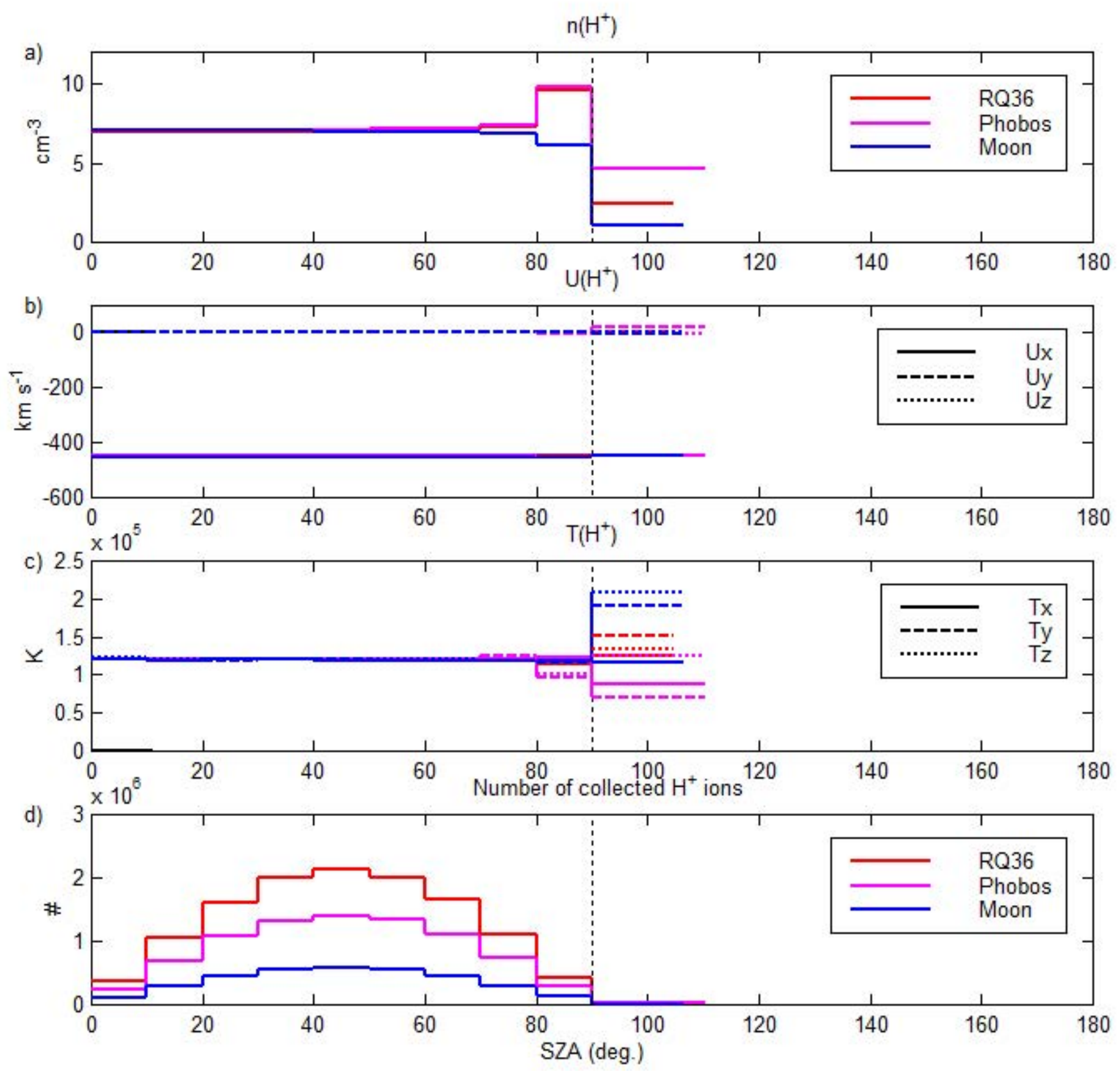
Fig. 4.

Electric potential for different SZA

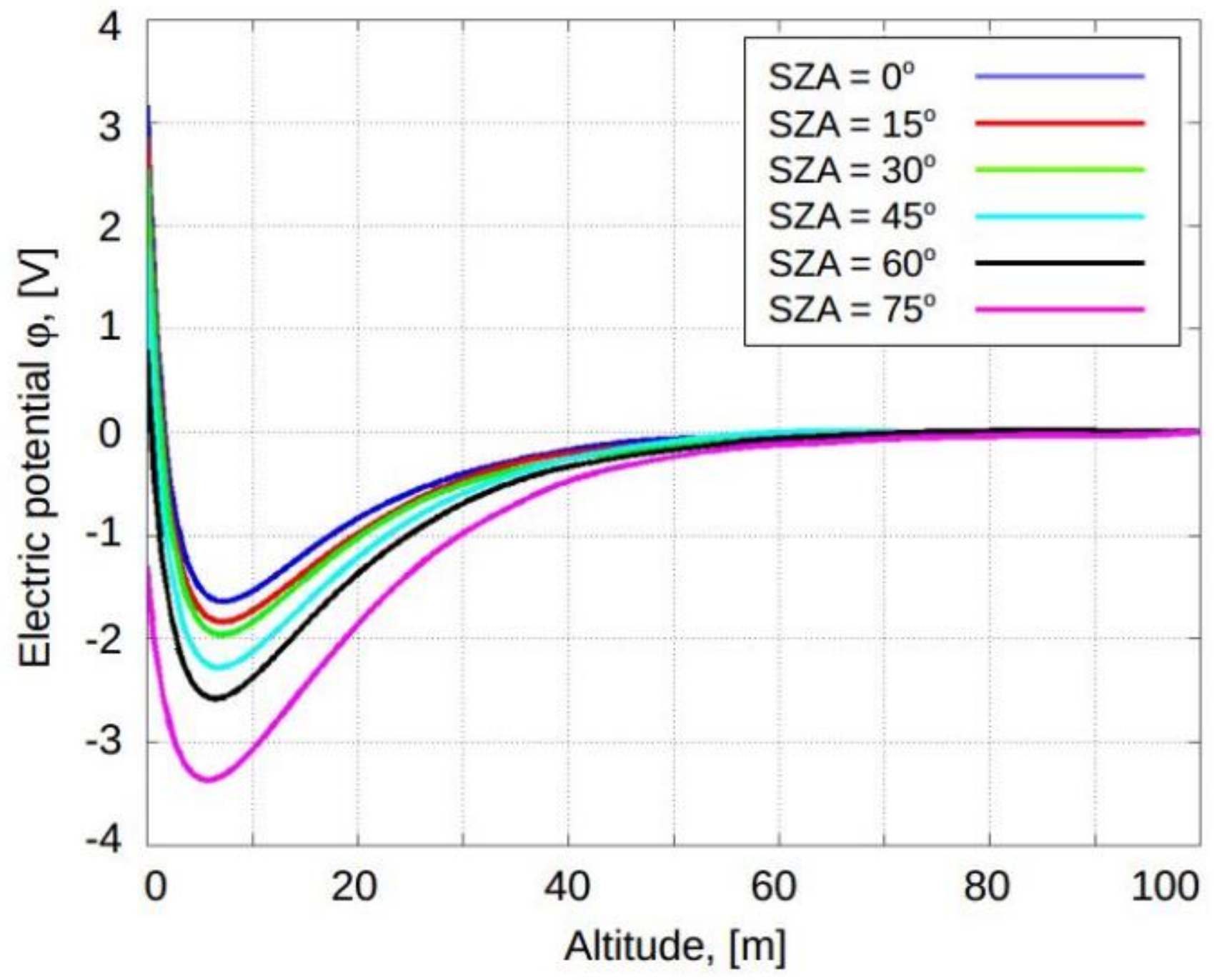


Fig. 5.

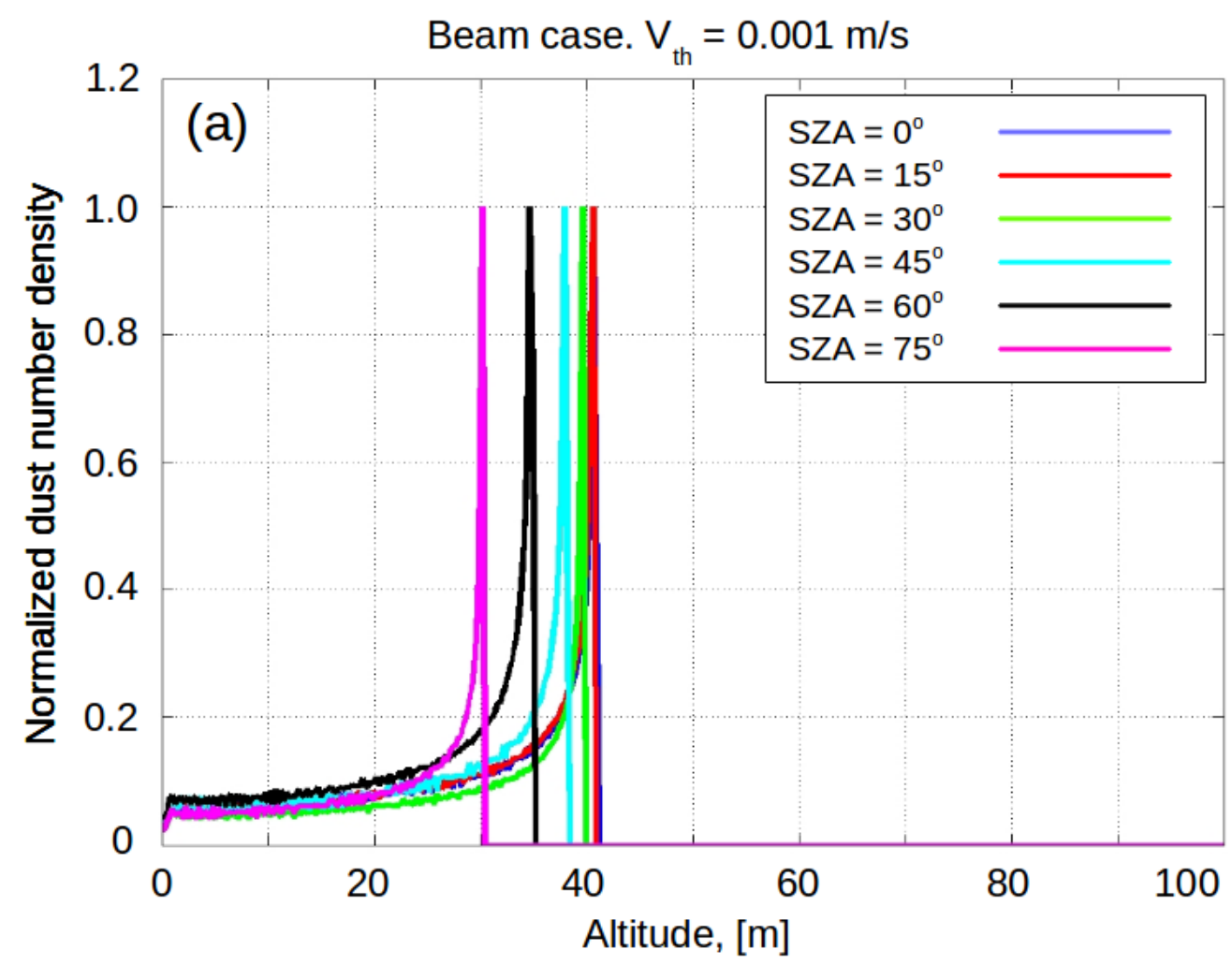

Hot case. $\mathrm{V}_{\text {th }}=2.0 \mathrm{~m} / \mathrm{s}$

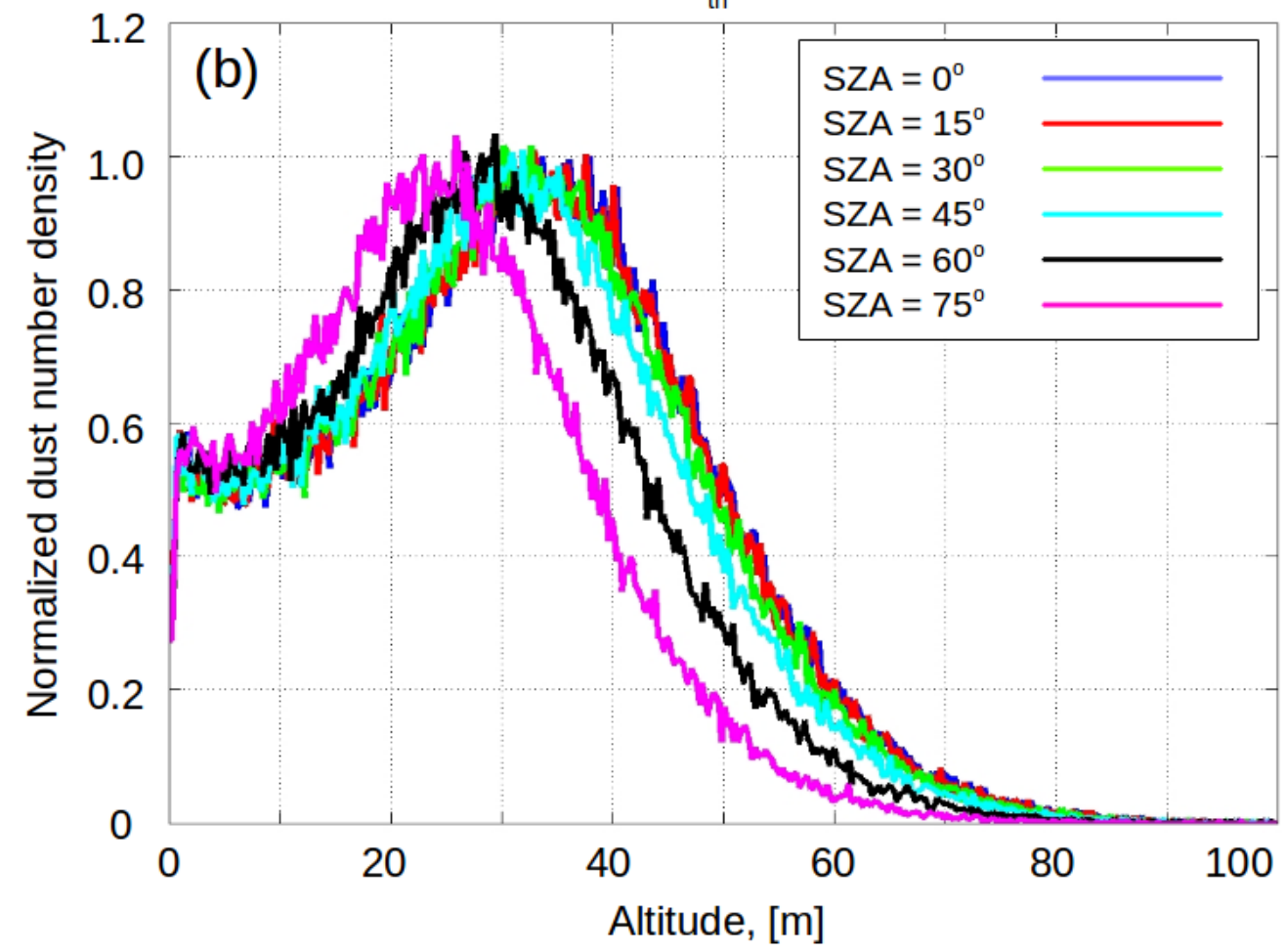




\section{Asteroid RQ36 (no surface charge)}

a) Run RQ36-1: $\mathrm{v}_{\mathrm{d}}=0 \mathrm{~m} / \mathrm{s} \mathrm{T}_{\mathrm{d}}=300 \mathrm{~K}, \mathrm{~m}_{\mathrm{d}}=3.7 \times 10^{9} \mathrm{amu}$, full surface source
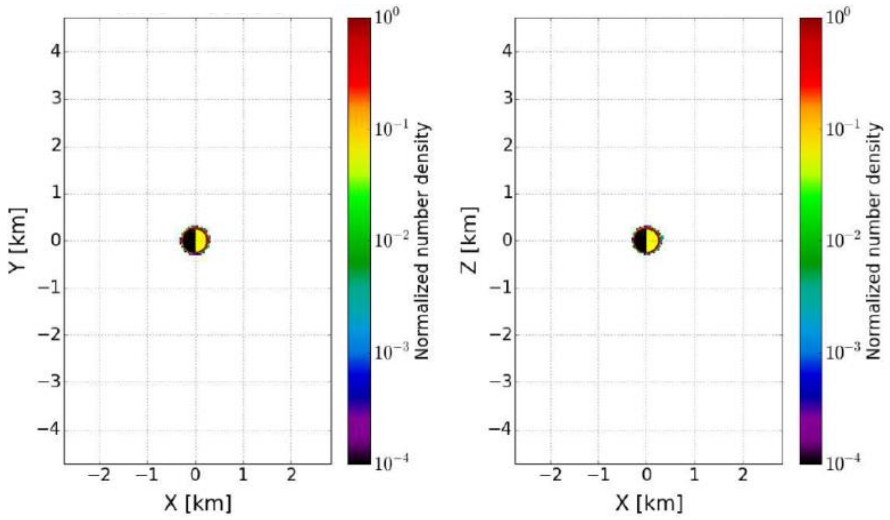

b) Run RQ36-2: $v_{d}=0.2 \mathrm{~m} / \mathrm{s} \mathrm{m}_{d}=3.7 \times 10^{9} \mathrm{amu}$, a point source $(R, R, R) / v 3$
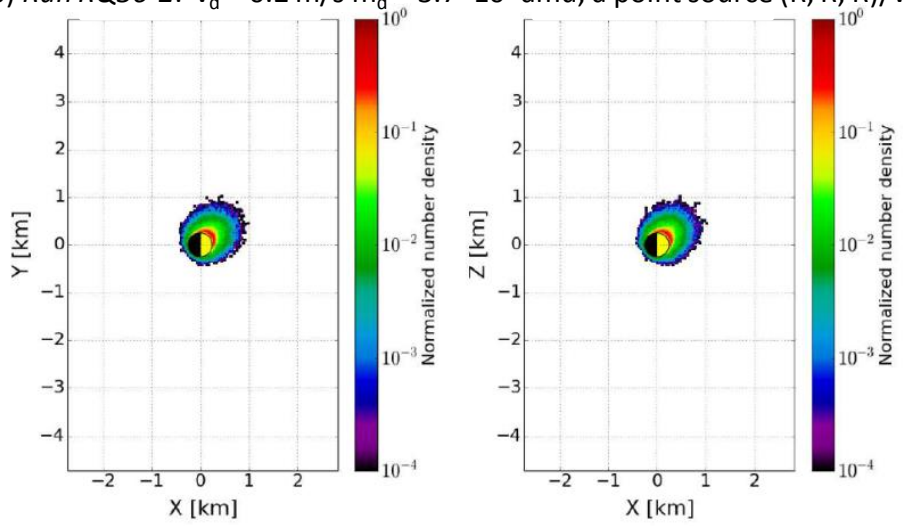

c) Run RQ36-3: $v_{d}=0.2 \mathrm{~m} / \mathrm{s} \mathrm{m}_{\mathrm{d}}=3.7 \times 10^{8} \mathrm{amu}$, a point source $(\mathrm{R}, \mathrm{R}, \mathrm{R}) / \mathrm{v} 3$
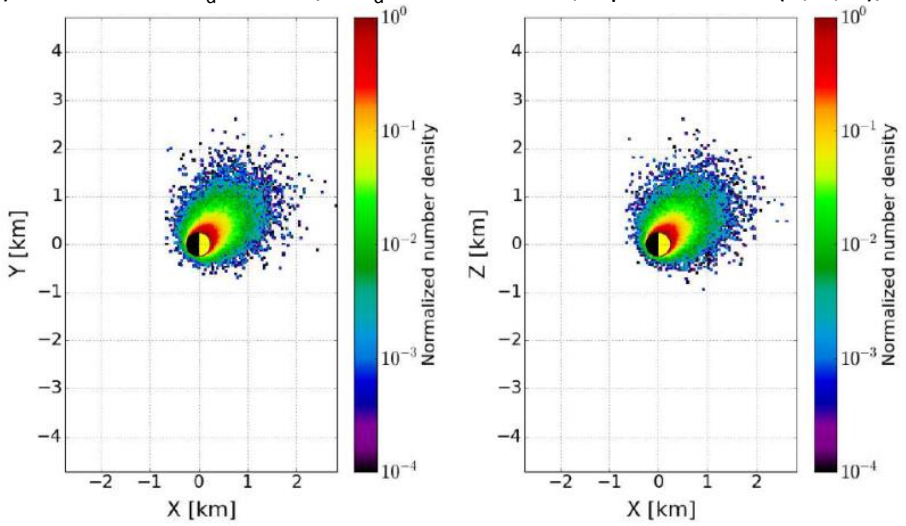

d) Run RQ36-4: $v_{d}=0.4 \mathrm{~m} / \mathrm{s} \mathrm{m}_{\mathrm{d}}=3.7 \times 10^{9} \mathrm{amu}$, a point source $(\mathrm{R}, \mathrm{R}, 0) / \mathrm{v} 2$
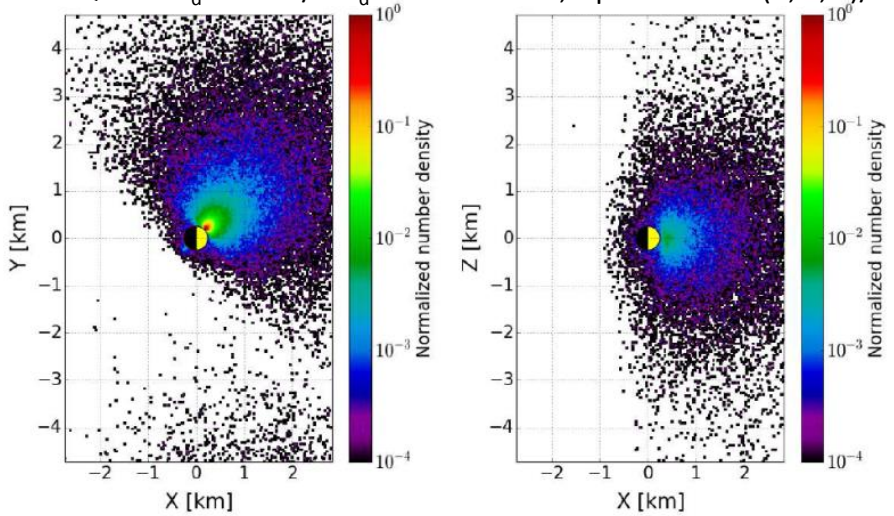


\section{Asteroid RQ36 (surface charge included)}

a) Run RQ36-5: $\mathrm{v}_{\mathrm{d}}=0 \mathrm{~m} / \mathrm{s} \mathrm{T}=300 \mathrm{~K}, \mathrm{~m}_{\mathrm{d}}=3.7 \times 10^{9} \mathrm{amu}$, full surface source
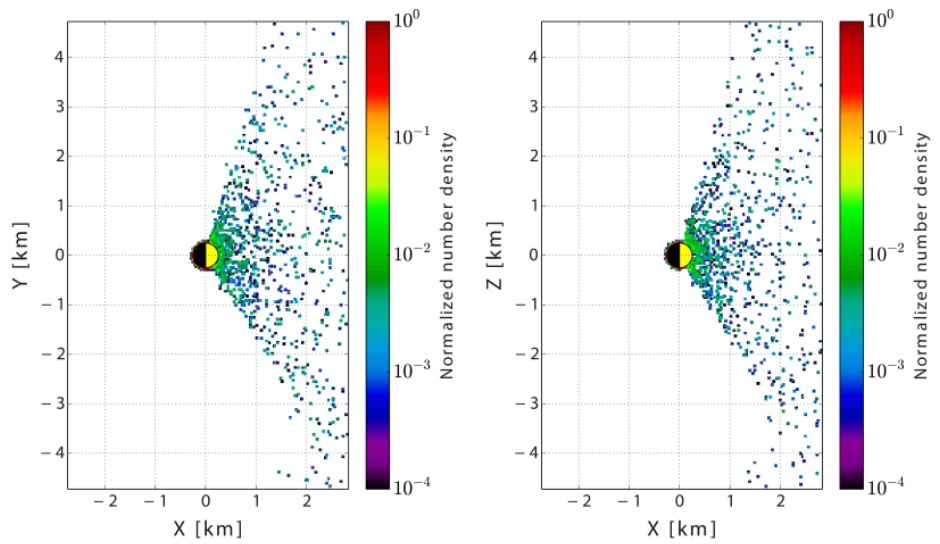

b) Run RQ36-6: $\mathrm{v}_{\mathrm{d}}=0.4 \mathrm{~m} / \mathrm{s} \mathrm{m}_{\mathrm{d}}=3.7 \times 10^{9} \mathrm{amu}$, a point source $(\mathrm{R}, \mathrm{R}, 0) / \mathrm{v} 2$
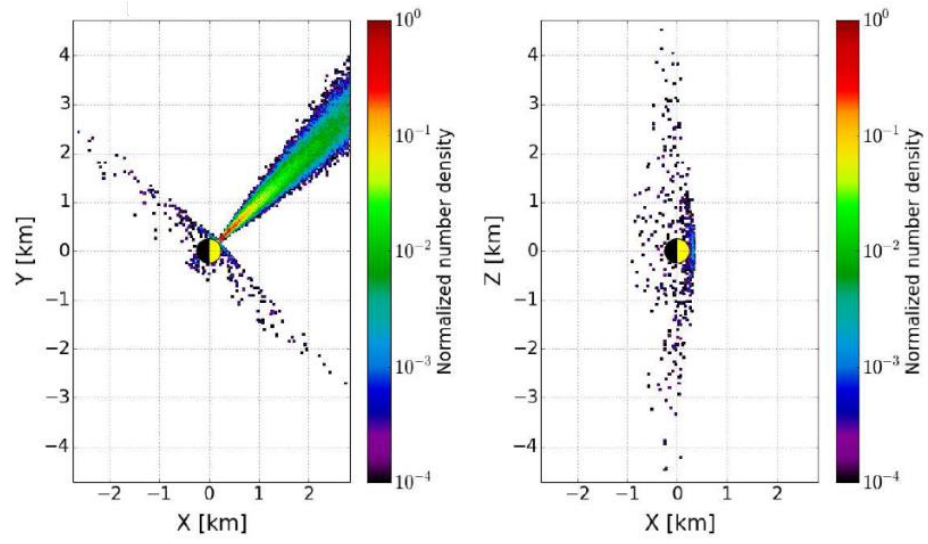


\section{Phobos}

a) Run Phobos- 1 : $\mathrm{v}_{\mathrm{d}}=0 \mathrm{~m} / \mathrm{s} \mathrm{T}_{\mathrm{d}}=300 \mathrm{~K}, \mathrm{~m}_{\mathrm{d}}=3.7 \times 10^{9} \mathrm{amu}$,

full surface source, no surface charging

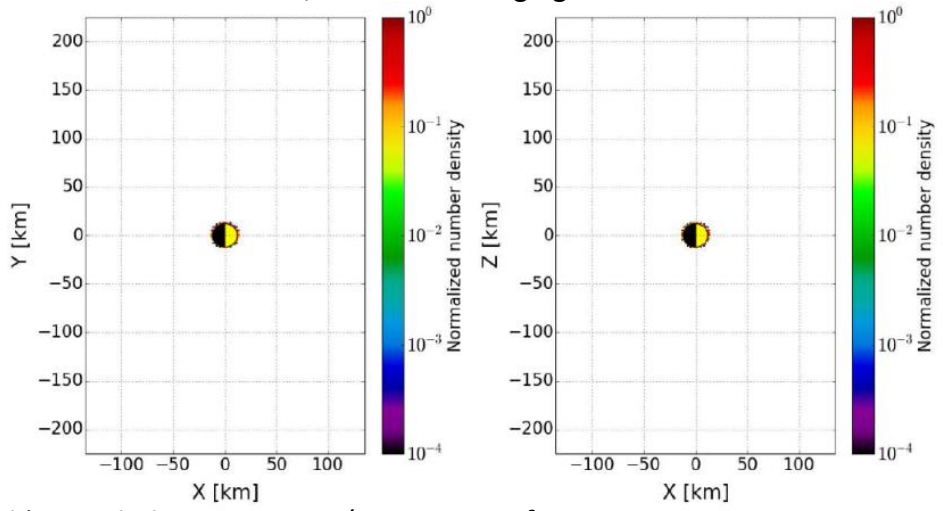

b) Run Phobos-2: $\mathrm{v}_{\mathrm{d}} \sim 12 \mathrm{~m} / \mathrm{s} \mathrm{m}_{\mathrm{d}}=3.7 \times 10^{9} \mathrm{amu}$,

a point source $(R, R, 0) / v 2$, no surface charging
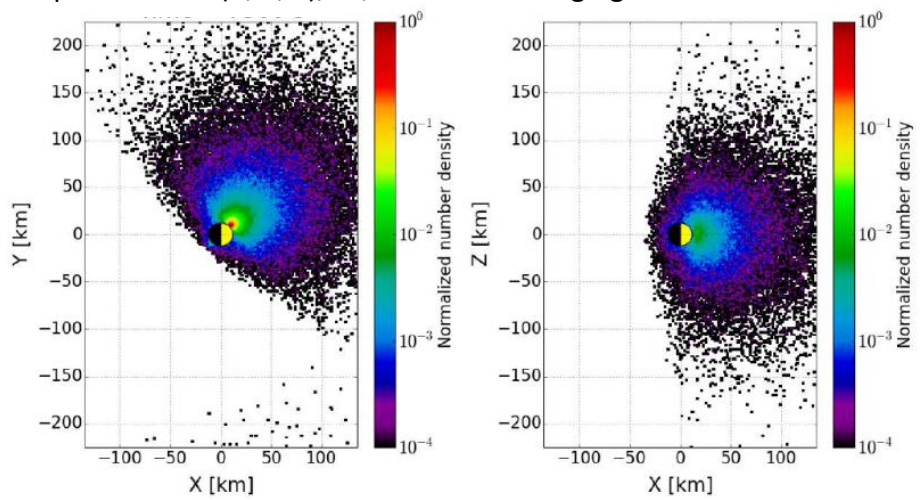

c) Run Phobos-3: $\mathrm{v}_{\mathrm{d}}=0 \mathrm{~m} / \mathrm{s} \mathrm{T}_{\mathrm{d}}=300 \mathrm{~K}, \mathrm{~m}_{\mathrm{d}}=3.7 \times 10^{9} \mathrm{amu}$,

full surface source, with surface charging
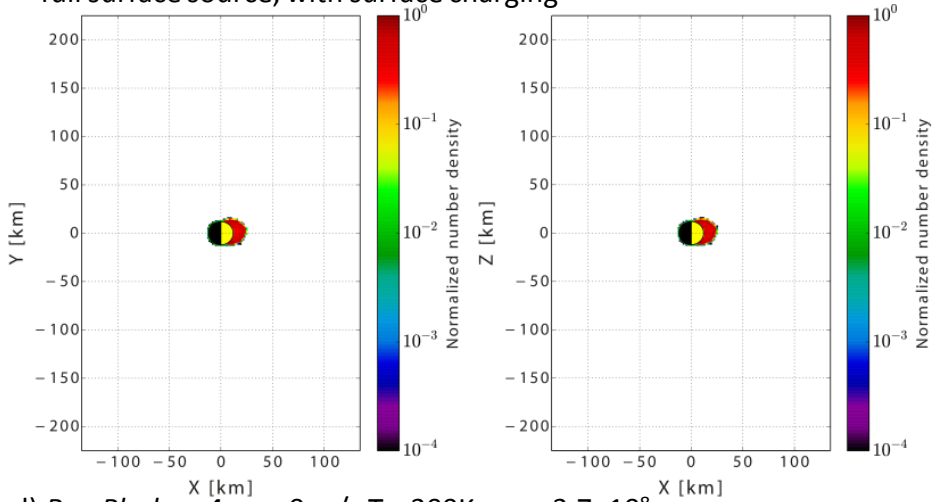

d) Run Phobos $-4: v_{d}=0 \mathrm{~m} / \mathrm{s} \mathrm{T}_{\mathrm{d}}=300 \mathrm{~K}, \mathrm{~m}_{\mathrm{d}}=3.7 \times 10^{8} \mathrm{amu}$,

full surface source, with surface charging
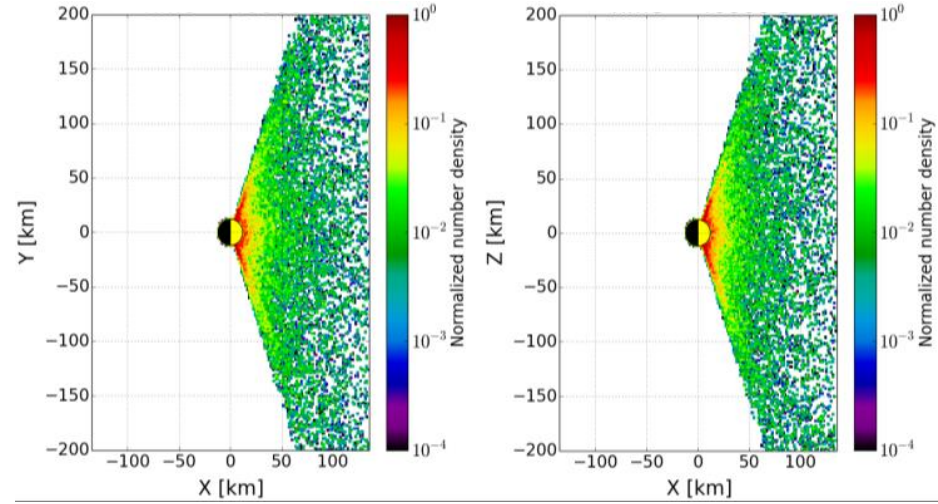
Fig. 9.

\section{The Moon}
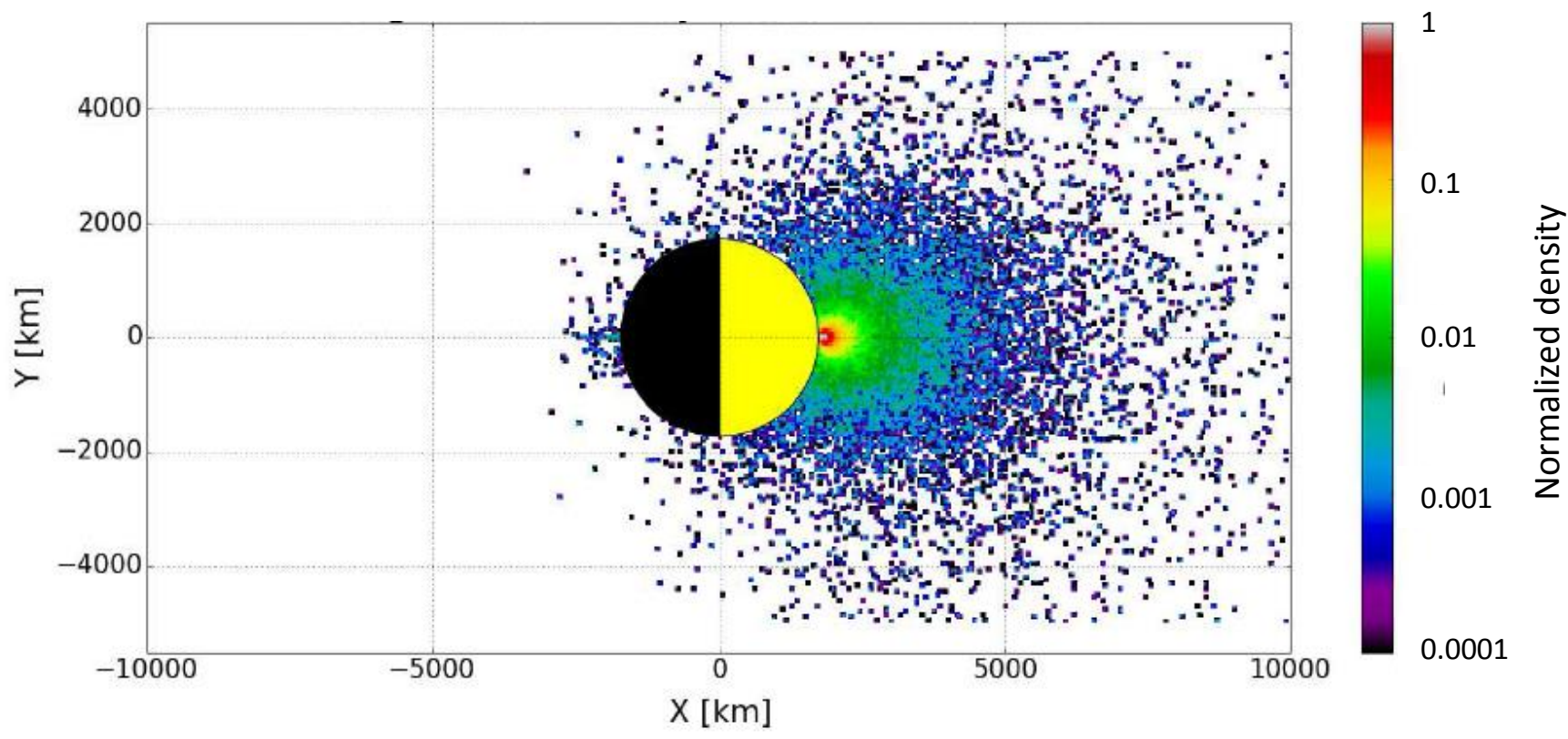

0.0001 
Fig. 10.

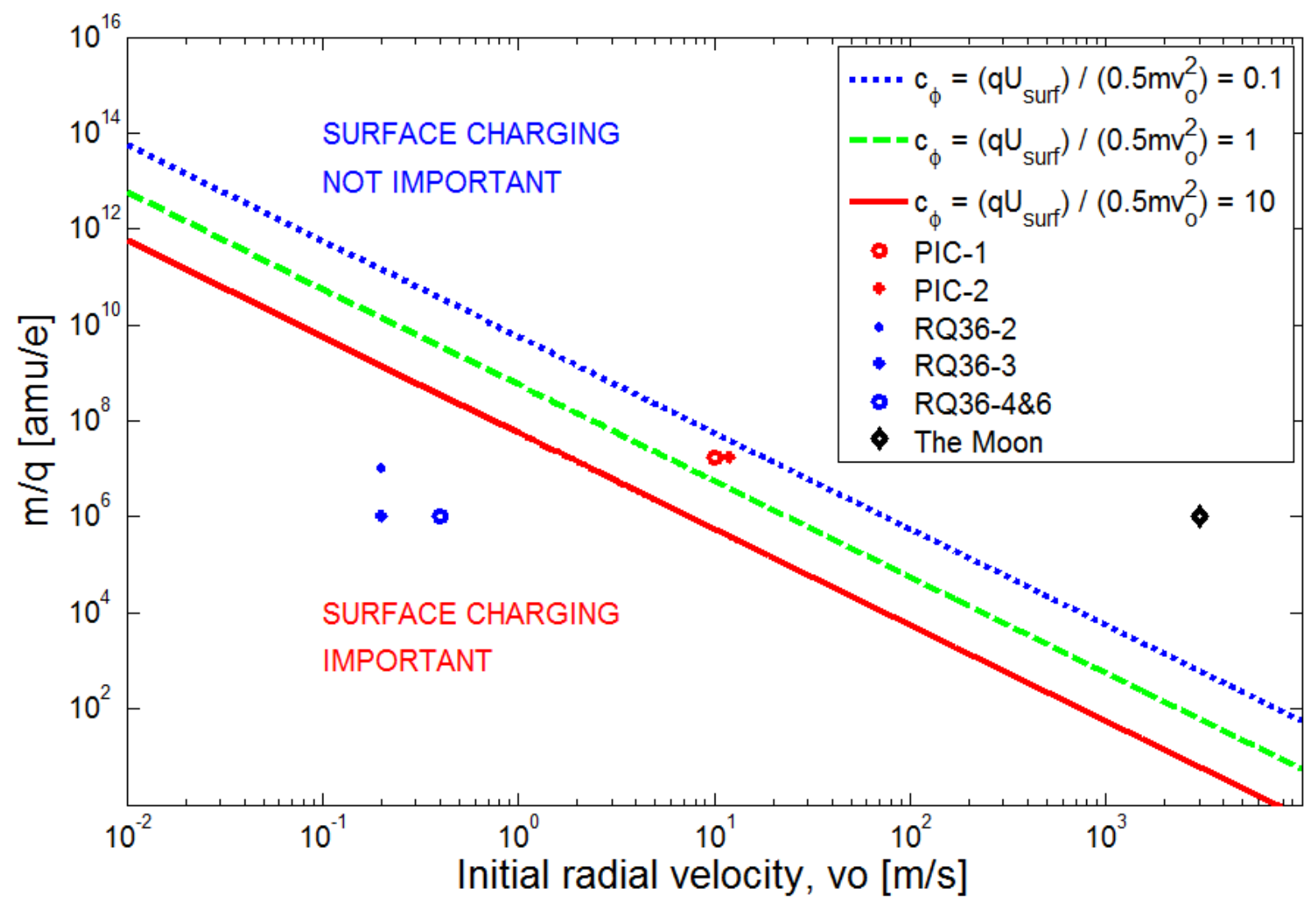

